\title{
Magnetic and mechanical buckling: Modified Landau theory approach to study phase transitions in micromagnetic disks and compressed rods
}

\author{
Sergey Savel'ev ${ }^{1}$ and Franco Nori ${ }^{1,2}$ \\ ${ }^{1}$ Frontier Research System, The Institute of Physical and Chemical Research (RIKEN), Wako-shi, Saitama, 351-0198, Japan \\ ${ }^{2}$ Center for Theoretical Physics, Center for the Study of Complex Systems, Department of Physics, University of Michigan, \\ Ann Arbor, Michigan, 48109-1120, USA \\ (Received 4 January 2004; revised manuscript received 14 June 2004; published 16 December 2004)
}

\begin{abstract}
Using the rigid magnetic vortex model, we develop a substantially modified Landau theory approach for analytically studying phase transitions between different spin arrangements in circular submicron magnetic dots subject to an in-plane externally-applied magnetic field. We introduce a novel order parameter: the inverse distance between the center of the circular dot and the vortex core. This order parameter is suitable for describing closed spin configurations such as curved or bent-spin structures and magnetic vortices. Depending on the radius and thickness of the dot as well as the exchange coupling, there are five different regimes for the magnetization reversal process when decreasing the in-plane magnetic field. The magnetization-reversal regimes obtained here cover practically all possible magnetization reversal processes. Moreover, we have derived the change of the dynamical response of the spins near the phase transitions and obtained a "critical slowing down" at the second order phase transition from the high-field parallel-spin state to the curved (C-shaped) spin phase. We predict a transition between the vortex and the parallel-spin state by quickly changing the magnetic field-providing the possibility to control the magnetic state of dots by changing either the value of the external magnetic field and/or its sweep rate. We study an illuminating mechanical analog (buckling instability) of the transition between the parallel-spin state and the curved spin state (i.e., a magnetic buckling transition). In analogy to the magnetic-disk case, we also develop a modified Landau theory for studying mechanical buckling instabilities of a compressed elastic rod embedded in an elastic medium. We show that the transition to a buckled state can be either first or second order depending on the ratio of the elasticity of the rod and the elasticity of the external medium. We derive the critical slowing down for the second-order mechanical buckling transition.
\end{abstract}

DOI: 10.1103/PhysRevB.70.214415

PACS number(s): $75.75+a, 46.32 .+x, 05.70 . F h$

\section{INTRODUCTION}

\section{A. Overview of magnetic microdots}

Recent achievements in nanotechnology now allow the fabrication of different arrays of small magnetic dots of various shapes and different interdot spacings. ${ }^{1-18}$ The size of these small magnetic dots range from several tens to several hundred nanometers in length and from a few to several tens of nanometers in thickness. Such dot arrays are potentially useful for memory elements, ${ }^{1}$ magnetic field sensors, ${ }^{19}$ and logic devices, ${ }^{2}$ among other applications. By using different experimental techniques, including magneto-optical Kerr effect, ${ }^{1,2,8,11-13}$ Lorentz transmission electron microscopy, ${ }^{6,7,10}$ and magnetic force microscopy, ${ }^{3-5,8,13,14}$ it has already been observed, that the magnetic vortex, $1,3,5-10$ parallel-spin state, ${ }^{1,8}$ and different realizations of curved spin states [also known as bent spin states, including the so-called C-phase (e.g. Refs. 6 and 10), and the S-phase (e.g., Ref. 10)] compete for the magnetization reversal process and the remanent state. For instance, different sequences of these magnetic states can contribute to the magnetization reversal process depending on the shape of the dots,,${ }^{6,7,15}$ dot sizes, ${ }^{7}$ interdot distances ${ }^{10-13}$ and dot arrangements. ${ }^{11,12}$ Also, the detailed spin configuration depends on small structural defects, surface roughness, ${ }^{16}$ and small variation of the shape of the dots. ${ }^{6}$

Recent micromagnetic simulations also suggest different types of spin arrangements. Depending on the dot shape, its aspect ratio, interdot distance, and the exchange length, observed spin configurations include out-of-plane (1,20-22 $^{1,}$ and in-plane $e^{23,24}$ vortices, the onion state, ${ }^{23,24}$ the $\mathrm{C}$ and $\mathrm{S}$ bent states, ${ }^{16,21-24}$ etc. However, there is still no general and clear understanding of how each one of these magnetic phases transforms into another one when the magnetic field changes. For instance, recent simulations ${ }^{22-24}$ pose the question of why, in some region of parameters, the vortex state does not contribute to the reversal magnetization process even though it provides the minimum energy at zero magnetic field. Another related unclear issue is how one magnetic spin configuration loses its stability and transforms into another one and what happens with the dynamical response of magnetic dots when the spin configuration changes near the phase boundaries.

The detailed investigation of how different spin arrangements evolve when changing the in-plane magnetic field for different sets of parameters is very time consuming computationally (e.g., Ref. 22) and can sometimes provide contradictory results (see, e.g., Ref. 16) depending on the computer codes used. Thus, it is very desirable to explore alternative ways to study this problem without the use of micromagnetic computer codes.

\section{B. Novel order parameters to describe magnetic and mechanical phase transitions}

Instead of the usual microscopic description of spin arrangements, we use a modified Landau-type ${ }^{25}$ theory, study- 
ing the general properties of the magnetic phase evolution in micromagnetic dots. Indeed, the most significant modern advance in our general understanding of equilibrium critical phenomena ${ }^{26,27}$ was preceded by the gradual realization that apparently dissimilar and unrelated phenomena (e.g., chemical, mechanical, optical, magnetic, superconducting and superfluid transitions) shared some commonalities near critical points. More recently, the focus has shifted to systems driven away from equilibrium (e.g., Refs. 28-31) or in metastable states, and to the search for common behaviors and trends near their phase transitions. For example, the mechanical stick-slip plastic effect, known as the Portevin-Le Châtelier effect (PLC), was studied in Ref. 28 by compressing Al$\mathrm{Mg}$ alloys in a very large deformation range, and the results interpreted from the viewpoint of phase transitions and critical phenomena. The experiments in Refs. 28 and 29 applied a generalized force, the externally imposed stress, on metallic samples that responded by generating intermittent bursts. This is the mechanical analog of applying an external magnetic field, another generalized force, to a spin configuration, and monitor its continuous and discontinuous response. The mechanical response was quantified by the rate of burst generation, $1 / \tau_{\text {bursts }}$, which can be seen as the order parameter for the mechanical stick-slip plastic effect observed there. ${ }^{28}$ This is the analog of the magnetization $M$ in a magnet, or the density of paired electrons in superconductors.

In order to develop our modified Landau-type theory for studying the phase transitions among different spin arrangements in micromagnetic dots, we restrict ourselves to circular flat dots with no interdot interactions, which is valid ${ }^{12}$ if the interdot distance is larger than the dot radius $R$. Moreover, we will utilize the commonly used "rigid" vortex model (see, e.g., Refs. 11, 20, 21, and 32), which describes different spin arrangements in terms of the displacement of the vortex core from the disk center. Namely, based on earlier works, ${ }^{33,34}$ several groups ${ }^{11,20,21,32}$ have assumed that the magnetic vortex core [Fig. 1(a)] moves away from the center of the disk, keeping the spin arrangement unchanged, when the magnetic field increases from zero to an "annihilation field" corresponding to the disappearance of the vortex. When the externally applied in-plane magnetic field decreases from some very high value, the center of the magnetic vortex can move from infinity [parallel-spin state [(sometimes called single-domain state $\left.\left.{ }^{35}\right)\right]$ towards the center of the disk with the vortex core still sitting outside the dot [Fig. 1(b)]-this describes the so-called C-phase ${ }^{11,21}$ since the spin arrangement inside the disk has a $\mathrm{C}$-shape configuration. Therefore, we will consider how these three magnetic phases (the vortex phase, the C-phase and the parallel-spin state) evolve when the in-plane magnetic field is varied.

Note that the rigid vortex model has been successfully used to obtain values of the applied magnetic fields for the annihilation and nucleation of a vortex ${ }^{11,20,21}$ in small micromagnetic disks. These results are consistent with both experiments and numerical simulations. Also, the rigid vortex model has been useful to study some dynamical properties of magnetic vortices. ${ }^{36}$ These works (e.g., Refs. 11, 20, 21, 32, and 36) almost prove the applicability of the rigid vortex model for describing the evolution of spin structures in an

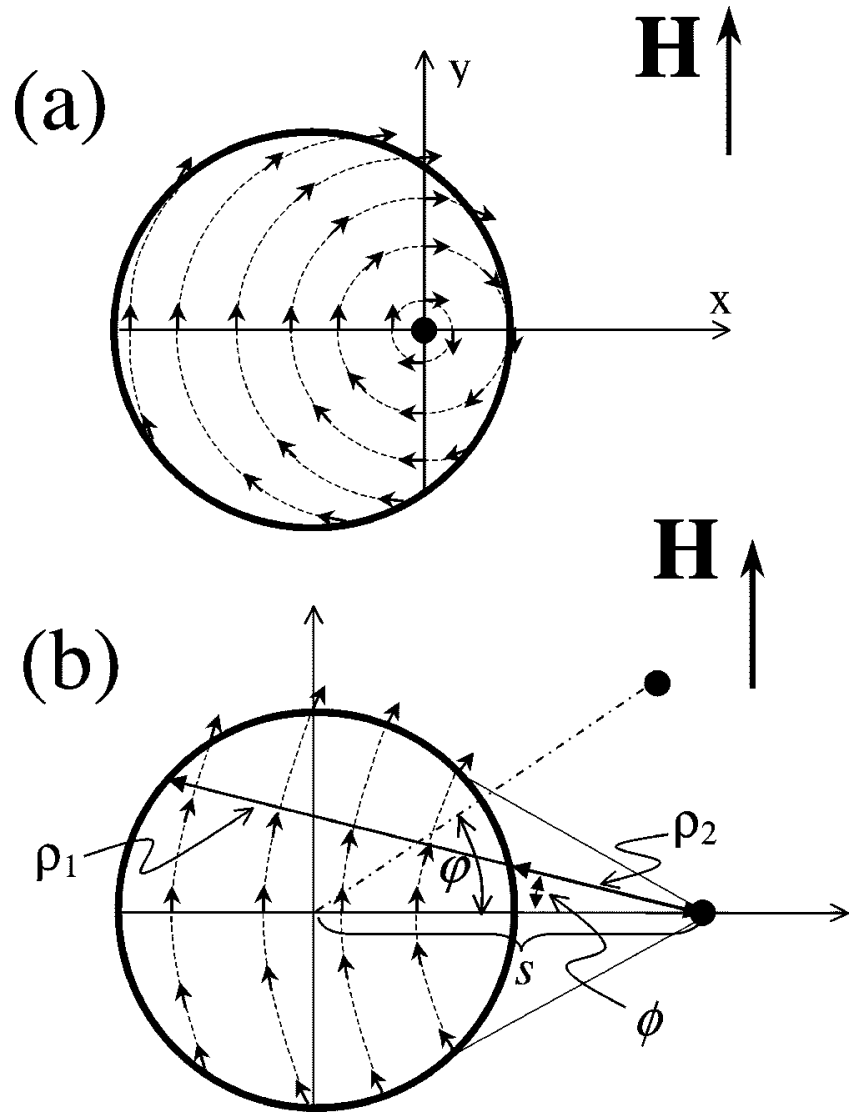

FIG. 1. (a) Schematic diagram of a magnetic vortex sitting inside the dot and shifted from the dot center because of an applied in-plane external magnetic field $H$. The coordinate system is centered at the vortex core. The $z$-axis is perpendicular to the surface of the dot and towards the reader. (b) The so-called C-phase or bent spin configuration is modeled as a magnetic vortex sitting outside the dot. For this case $(s>1)$, the origin of the coordinate system is located at the dot center. The angle $\varphi$ describes the possible rotation of the vortex around the dot center. The angle $\phi$ and the $\phi$-dependent distances $\rho_{1}(\phi)$ and $\rho_{2}(\phi)$ are used in Appendix A in order to derive the exchange and Zeeman energies.

array of micromagnetic dots. Of course this model is phenomenological, but has been verified via micromagnetic simulations and experiments. In addition, this model provides a great advantage: the spin system can be described by only one degree of freedom - the distance $s$ between the magnetic vortex and the dot center. This makes the problem analytically tractable.

Here, we introduce a novel order parameter, which is not the usual average magnetization used for description the magnetic phase transition ${ }^{25}$ in bulk materials, but the inverse distance $\psi=1 / \mathrm{s}$ between the center of the disk and the vortex center. Expanding the energy of the rigid vortex with respect to this new order parameter, we derive a modified Landautype theory describing magnetic states for micromagnetic disks. Interestingly, the order parameter introduced here exhibits some analogies with other systems, including elastic and plastic deformation transitions. ${ }^{28}$ For the micromagnetic disk case, the order parameter 


$$
\psi=\frac{1}{s}
$$

describes transitions among metastable states, while for plastic stick-slip deformations ${ }^{28}$

$$
\psi_{p}=\frac{1}{\tau_{\text {bursts }}}
$$

is used to describe a temporal order parameter. Near the dynamic critical points, the order parameter $\psi_{p}=1 / \tau_{\text {bursts }}$ of the PLC effect exhibits large fluctuations, and "critical slowing down," in analogy with the critical slowing down predicted here for micromagnetic disks. Note that critical slowing down is a quite general dynamical property near second order phase transitions, and can manifest itself in condensed matter, cosmology, and other areas. ${ }^{37}$

We have found a much closer mechanical analog by comparing the transition between the parallel-spin state and curved spin state (magnetic buckling) with the known mechanical buckling instabilities (see, e.g., Chap. II of Ref. 38). This analogy is schematically shown in Fig. 2. Using the approach we develop for the micromagnetic disk case, we study the second and first order buckling transitions for a compressed rod placed in an elastic medium. ${ }^{38}$ Our approach is more general than other approaches used for studying the second-order buckling transition. ${ }^{39,40}$

Note that recently there is a growing interest in the buckling of small rods in the context of, e.g., mechanical properties and stability of carbon nanotubes, ${ }^{41-45}$ multilayers, ${ }^{46}$ biopolymers, ${ }^{47,48}$ DNA, ${ }^{49,50}$ and fracture. ${ }^{51,52}$ A nice modern introduction to the application of buckling to pattern formation in physical and biological systems can be found in Ref. 53. Also, the mechanical bending and buckling of microrods is currently explored for novel applications in nanomechanics (e.g., Ref. 54), as well as for quantum detection and information processing. ${ }^{55-58}$

\section{Summary of results}

Although the simple rigid vortex model cannot describe very complicated spin arrangements in magnetic dots (e.g., "S"-shaped spin arrangements), the magnetization-reversal regimes obtained here cover practically all possible magnetization reversal processes. Depending on the radius and thickness of the dot, as well as the exchange coupling, there are five different regimes (see Fig. 3) for the magnetization reversal process when decreasing the externally applied inplane magnetic field.

(1) For large radius of the microdisk: when lowering the magnetic field from an initial high value, the parallel-spin magnetic state of the microdisk becomes metastable and then unstable for a certain value of the (nucleation) external magnetic field $H_{n}$ and transforms to the vortex phase (via a first order phase transition). When the magnetic field changes polarity and increases in absolute value, the vortex phase first becomes metastable and afterwards it transforms (another discontinuous transition) to a parallel-spin state at a certain negative value, $-H_{a n}$, of the magnetic field.

(2) For intermediate disk radius: the bent or C-shaped spin arrangement (the $\mathrm{C}$ phase) mediates the transition between the parallel-spin state and the vortex state. Moreover, the transition from the parallel-spin state to the C-phase (and vice versa) is a continuous second-order transition, while the C-phase transforms to the vortex state via a discontinuous transition.

(3) For smaller disk radius: the vortex state does not participate in the magnetization reversal process while the parallel-spin state first transforms to the C-phase, which survives up to zero magnetic field. When the magnetic field changes its polarity, the $\mathrm{C}$-phase rotates first and then transforms to a parallel-spin state at a high negative external field. This rotation of the $\mathrm{C}$-phase when the polarity changes corresponds to a Goldstone mode moving along the minima of a hat-shaped potential. This rotation, like all Goldstone modes, costs either zero or little energy.

(4) For even smaller disk radius: the parallel-spin state rotates as a whole at zero magnetic field. Nevertheless, the vortex phase is stable or metastable at low magnetic fields in the third and fourth reversal magnetization regimes described above; this results in the initial (virgin) magnetization process.

(5) For even smaller radius: the parallel-spin phase is the only state having an energy minimum for any value of the external magnetic field. The vortex and $\mathrm{C}$ phase are not accessible.

The dynamical response of the spin configurations changes drastically at the phase transitions, producing "critical slowing down" at the second-order phase transition between the parallel-spin and the $C$ phase. We want to stress that the obtained physical scenarios are related to the spontaneous symmetry breaking at the transitions between the parallel-spin state and the bent or vortex states and, therefore, these results are more general than the rigid vortex model itself. Moreover, our predicted "critical slowing down" has already been verified in micromagnetic simulations: ${ }^{59}$ this confirms the second order phase transition derived here between the parallel-spin phase and the C-phase. Hence, this physical picture could be generalized to some extent to much more complicated spin configurations in the circular thin dots, ${ }^{16,22,23}$ and certainly can be used as a guide to look for novel ways to control spin configuration in magnetic dots. It can also be generalized to micromagnetic disks exposed a transverse or out-of-plane applied magnetic field.

Using the very physical analogy [Figs. 2(a)-2(d)] between the "straight-to-bent rod" Euler buckling instability and the "parallel-to-curved" spin configuration phase transition in micro-magnetic disks, we have extended our modified Landau-type theory approach [Figs. 2(e)-2(h)] for an elastic rod embedded in an elastic medium. We found that the buckling transition can be either first or second order depending on the ratio of the rod and external-medium elasticity. For the case of the second order buckling, we predict that the dynamical response exhibits a "critical slowing down."

It is important to stress that the results obtained for both magnetic and mechanical buckling do not seem to be modeldependent since they are based on the general symmetrybreaking mechanism of phase transitions. 


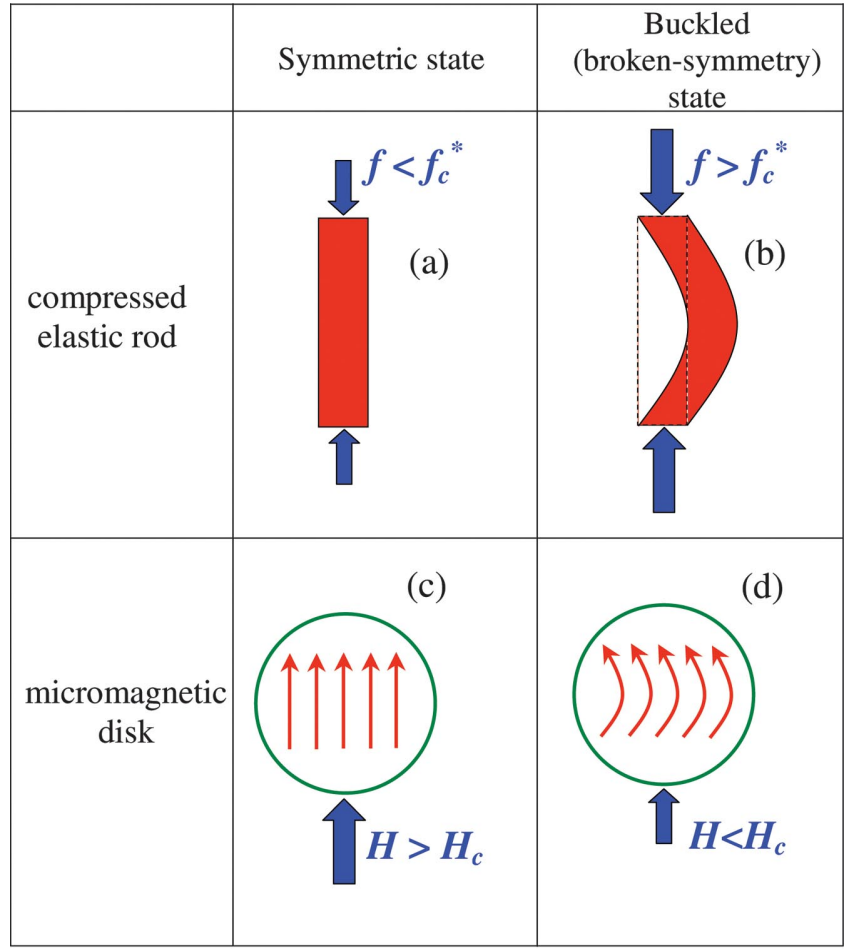

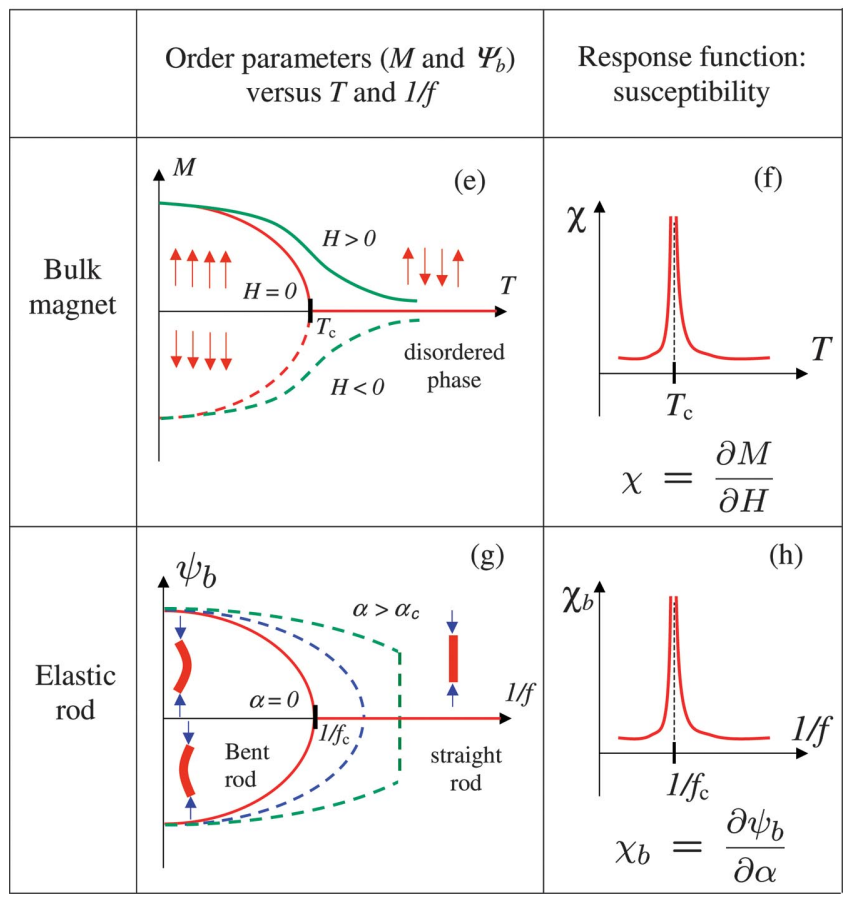

\section{ENERGY OF SPINS IN A DOT IN A MAGNETIC FIELD}

The energy of a spin system in a dot subject to an external magnetic field contains three contributions: (i) the exchange energy, describing nearest-neighboring spin interactions; (ii) the magnetostatic energy, attributed to the magnetic interaction of spins; and (iii) the Zeeman energy, which takes into consideration the interaction of spins with the external magnetic field. In the continuous approximation, the exchange
FIG. 2. (Color online) (a-d) When increasing an externally applied force $f$ on a compressed bar, it eventually changes from a staight configuration (a) to a bent one (b) via a (first or second order) buckling transition. This Euler Buckling instability Ref. 38 is an example of spontaneous symmetry breaking (i.e., the bar can bend either facing left or right). We study this here as a phase transition. Here we also consider a magnetic analog of this mechanical spontaneous symmetry breaking transition. When decreasing the value of an externally applied magnetic field $H$, the spin arrangement inside a micromagnetic disk changes from a "straight" or "perfectly aligned" parallel-spin configuration (c) to a "bent" or C-shaped magnetization (d) with the spin configuration following a curved or bent shape. $1 / \mathrm{H}$ plays the role of a generalized force. This is an example of a magnetic spontaneous symmetry breaking transition (e.g., the C-phase can face either to the left or to the right). This is studied here as a phase transition by expanding the magnetic energy of the dot. The order parameters ( $M$ and $\psi_{b}$ ) versus "generalized forces" are schematically shown in $(e, g)$. The effective response function, or susceptibility, for the standard ferromagnetic-paramagnetic phase transition and the buckling phase transition are compared in (f, h). (e) For zero externally applied magnetic field $H$, the magnetization $M$ is zero in the paramagnetic phase and starts to continuously increase in the ferromagnetic phase when lowering the temperature below $T_{c}$. As schematically shown in (e), an applied magnetic field $H \neq 0$ smears out the $M(T)$ phase transition, resulting in a nonzero magnetization in the paramagnetic phase. (f) The magnetic susceptibility $\chi=\partial M / \partial H$ has a peak at $T_{c}$. $(\mathrm{g})$ The transverse buckling amplitude $\psi_{b}(f)$ increases continuously when the compression force $f$ exceeds a critical value $f_{c}^{*}$, for small values of the elasticity $\alpha$ of the external medium. However, the continuous $\psi_{b}(f)$ transition becomes a discontinuous (first order) transition for higher values of the elasticity $(\alpha$ $>\alpha_{c}$ ). (h) The buckling of the rod becomes very sensitive to changes in the parameters when it is near $f_{c}^{*}$, exhibiting critical slowing down and a peaked effective susceptibility $\chi_{b}=\partial \psi_{b} / \partial \alpha$. A closer analog of the external magnetic field $H$ is an external force $f_{\perp}$ perpendicular to the rod and applied near its center. Note that $f_{\perp}$ buckles the rod while $\alpha$ suppresses its buckling. More precisely, sufficiently large values of either $f_{\perp}$ or $f$ buckle the rod, when $\alpha$ is sufficiently weak. If $\alpha \rightarrow \infty$, then $f$ and/or $f_{\perp}$ will not be able to buckle the rod. 

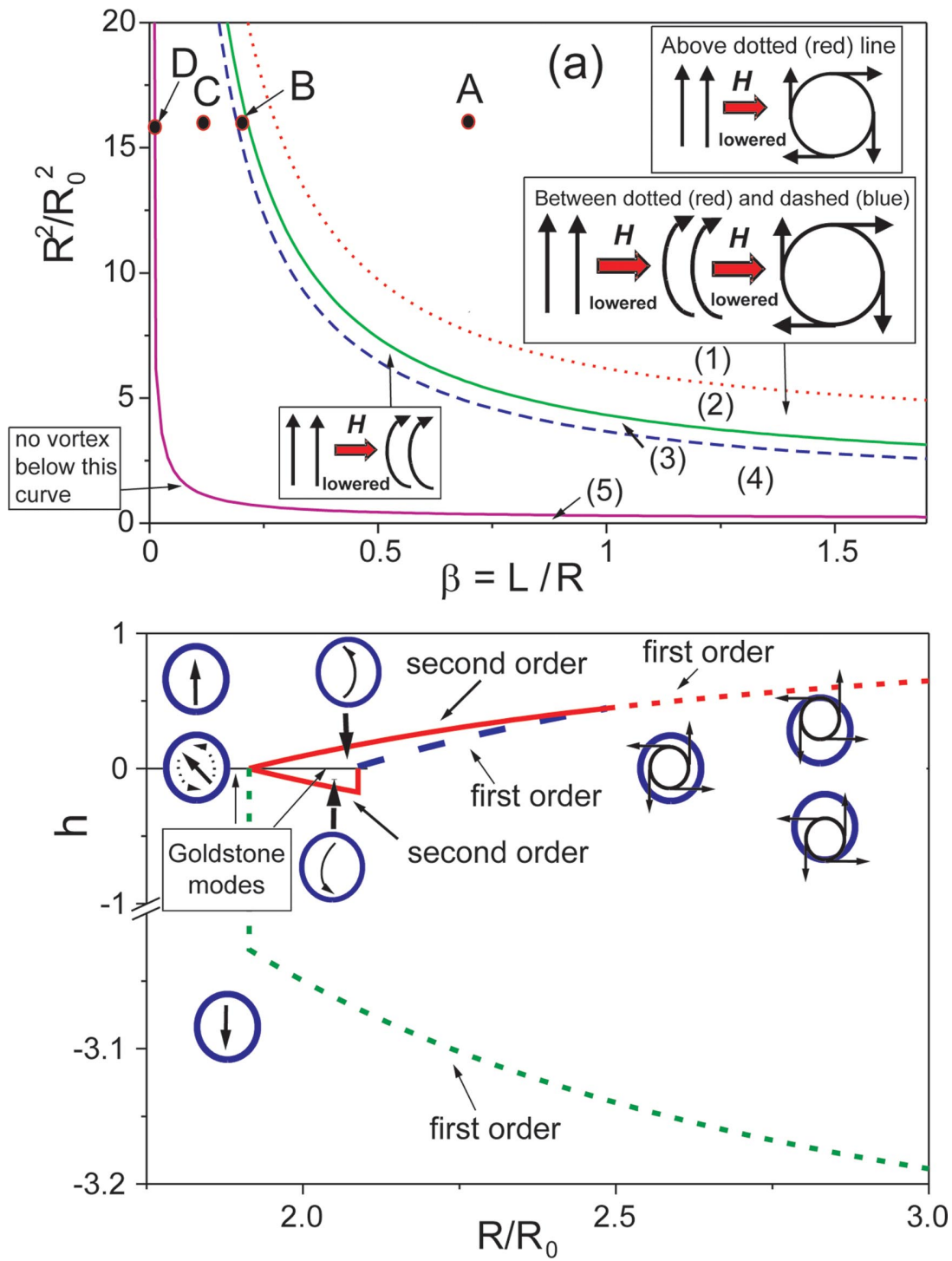

FIG. 3. (Color online) (a) Phase diagram showing the domains of parameters for the different sequences of transitions among three stable and metastable spin configurations inside a micromagnetic disk. The parameters are the square of the reduced radius $R^{2} / R_{0}^{2}$ versus the aspect ratio $\beta=L / R$. Above the dotted top curve, the parallel spin state, which exists for high applied in-plane magnetic fields, discontinuously transforms to a magnetic vortex sitting inside the disk. This is because the large radius of the microdisk can easily accommodate a vortex inside. For parameter values between the dotted and continuous curves (e.g., for smaller disk radius at a fixed $\beta$ ), a second-order phase transition from the parallel spin configuration to the C-phase occurs first. Upon further lowering the in-plane field, the $\mathrm{C}$ phase abruptly transforms to a vortex sitting inside the disk. Between the dashed and continuous curves, the C-phase survives down to $h=H / M_{s}=0$, and rotates as a whole when the magnetic field changes its polarity. This rotation costs little or no energy and it is a Goldstone mode (Refs. 61-66). Below the dashed curve, the magnetization reversal process proceeds via a rotation of the parallel-spin state as a whole at $h=0$. Even though the vortex state does not contribute to the magnetization reversal process below the continuous curve, the magnetic vortex is stable or metastable at low magnetic fields above the continuous curve located at the bottom of the diagram. In practice, the stable or metastable vortex states below the continuous curve cannot be reached besides at high temperatures or when $H$ changes suddenly. Below this continuous bottom curve, the magnetic vortex does not correspond to an energy minimum and it is unstable for any value of $h$. (b) Phase diagram plotted on the plane [magnetic field $=(\text { generalized force })^{-1}=h=H / M_{s}$, reduced radius $=R / R_{0}$ ]. This shows magnetization reversal processes for a fixed aspect ratio $\beta=L / R=1$ when lowering the applied magnetic field $h$ from a high value $h>h_{c}$. Dashed (solid) lines correspond to first-(second-) order phase transitions. The short black segment at the top left corner corresponds to Goldstone modes, where the magnetization can be rotated with zero or little energy cost. For the case of increasing $h$ from a high negative value $h<-h_{c}$, the diagram is inverted with respect to the $h=0$ axis. In order to construct this diagram, we use Eqs. (18) and (B4) and the criterion $\psi=1$ for penetrating a magnetic vortex (transition from the C-phase to the vortex state). 
TABLE I. Comparison between the usual Landau approach to study magnetic phase transitions and the modified version studied here.

\begin{tabular}{|c|c|c|}
\hline & Standard approach & Our approach used here \\
\hline Order parameter & Magnetization $M$ & $\begin{array}{c}\psi=1 / s= \\
(\text { disk-vortex distance })^{-1}\end{array}$ \\
\hline $\begin{array}{l}\text { High } T \text { or } \\
\text { high } H \text { phase }\end{array}$ & $\begin{array}{c}M=0 \\
(\text { high } T \text { ) }\end{array}$ & $\begin{array}{c}\psi=0 \text { (parallel-spin phase) } \\
(\text { high } H)\end{array}$ \\
\hline $\begin{array}{l}\text { Low } T \text { or } \\
\text { low } H \text { phase }\end{array}$ & $\begin{array}{c}M \neq 0 \\
(\text { low } T)\end{array}$ & $\begin{array}{c}\psi \neq 0(\mathrm{C} \text { or vortex phase }) \\
(\text { low } H)\end{array}$ \\
\hline $\begin{array}{l}T=0 \text { or } \\
H=0 \text { phase }\end{array}$ & $\begin{array}{c}M=M_{s} \\
(T=0)\end{array}$ & $\begin{array}{c}\text { For certain parameter values } \\
\qquad \begin{array}{c}\psi=\infty(\text { vortex centered } \\
\text { in the dot center at } H=0)\end{array}\end{array}$ \\
\hline Energy [order parameter] & $F_{L}[M]=F_{L 0}+a M^{2}+b M^{4}-H M$ & $F[\psi]=F_{0}+A \psi^{2}+B \psi^{4}+\mathcal{O}\left(\psi^{6}\right)$ \\
\hline $\begin{array}{l}\text { Energy expansion } \\
\text { coefficients }\end{array}$ & $\begin{array}{c}T \text {-dependent, } \\
\text { phenomenological }\end{array}$ & $\begin{array}{c}H \text {-dependent, } \\
\text { analytically derived }\end{array}$ \\
\hline $\begin{array}{l}\text { Stability of the phases } \\
\text { determined by }\end{array}$ & $\begin{array}{l}\text { Truncated series expansion of } \\
\qquad F_{L}[M]\end{array}$ & $\begin{array}{c}\text { Full expression of } \\
w[\psi](\text { at all orders in } \psi)\end{array}$ \\
\hline $\begin{array}{l}\text { Odd powers are } \\
\text { zero because of }\end{array}$ & $\begin{array}{c}\text { symmetry } \\
F_{L}[M]=F_{L}[-M]\end{array}$ & $\begin{array}{l}\text { Analytically derived; this already satisfies } \\
\text { the symmetry restriction } F[\psi]=F[-\psi]\end{array}$ \\
\hline Coefficient of $\psi^{2}$ or $M^{2}$ & $a \propto\left(T-T_{c}\right)$ & $A \propto\left(h-h_{c}\right)$ \\
\hline Coefficient of $\psi^{4}$ or $M^{4}$ & $b=\mathrm{const}>0$ & $B(h, C, R, L) \gtrless 0$ \\
\hline 1st order transition & requires cubic term $\propto M^{3}$ & $A$ changes sign when $B<0$ \\
\hline 2nd order transition & $a$ changes sign and $b>0$ & $A$ changes sign when $B>0$ \\
\hline $\begin{array}{l}\text { Order parameter } \\
\text { near } T_{c} \text { or } h_{c}\end{array}$ & $M \propto\left(T_{c}-T\right)^{1 / 2}\left(\right.$ near $\left.T_{c}\right)$ & $\psi \propto\left(h_{c}-h\right)^{1 / 2}\left(\right.$ near $\left.h_{c}\right)$ \\
\hline $\begin{array}{l}\text { Susceptibility } \\
\text { near } T_{c} \text { or } h_{c} \\
\end{array}$ & $\begin{array}{c}\partial M(T) / \partial H \propto\left|T-T_{c}\right|^{-1} \\
\left(\text { near } T_{c}\right)\end{array}$ & $\begin{array}{c}\partial \psi(h) / \partial T \propto\left|h-h_{c}\right|^{-p} \\
\left(\text { near } h_{c}\right)\end{array}$ \\
\hline $\begin{array}{l}\text { Critical slowing down } \\
\text { near } T_{c} \text { or } h_{c}\end{array}$ & $\begin{array}{c}\delta M=\text { deviation from equilibrium } \\
\delta M(t) \propto \exp \left(-t / \tau_{m}\right) \\
\tau_{m}=\left(T_{c}-T\right)^{-1}, \quad T<T_{c} \\
\tau_{m}=\left(T-T_{c}\right)^{-1}, \quad T>T_{c}\end{array}$ & $\begin{array}{c}\delta \psi=\text { deviation from equilibrium } \\
\delta \psi(t) \propto \exp (-t / \tau) \\
\tau=-\eta B^{2} / A^{3}=512 \eta B^{2} /\left(h_{c}-h\right)^{3}, \quad h<h_{c} \\
\psi=\left(2 A t / \eta+\psi^{-4}(t=0)\right)^{-1 / 4}, \quad h>h_{c}\end{array}$ \\
\hline
\end{tabular}

magnetic energy $W_{m}$ is determined by the surface magnetic charges $^{20,21,32}$ as

$$
W_{m}=\frac{1}{2} \int d S \int d S^{\prime} \frac{\sigma(\boldsymbol{r}) \sigma\left(\boldsymbol{r}^{\prime}\right)}{\left|\boldsymbol{r}-\boldsymbol{r}^{\prime}\right|},
$$

where $\sigma=\boldsymbol{M} \cdot \boldsymbol{n}$ is the magnetic charge density with unit vector $\boldsymbol{n}$ directed outside of the dot surface, while $\boldsymbol{r}$ and $\boldsymbol{r}^{\prime}$ are the positions of the elements of the surface. Here, the integration has to be performed over the dot surface. The Zeeman energy $W_{H}$ has the usual form,

$$
W_{H}=-\int_{V} d^{3} \boldsymbol{r} M(\boldsymbol{r}) \cdot \boldsymbol{H}
$$

with integration over the volume of a dot.

\section{RIGID VORTEX MODEL}

In order to obtain the energy of the vortex sitting either inside or outside the circular dot we need to know the magnetization as a function of the vortex position. In the rigid vortex model, the magnetization inside the dot is defined by the solution: ${ }^{21,34} m_{\rho}=0, m_{\phi}= \pm \sin \vartheta(\rho)$, and $m_{z}= \pm \cos \vartheta(\rho)$, where $\tan [\vartheta(\rho) / 2]=\rho / \tilde{b}$, with vortex core radius $\tilde{b}$. Here $m_{\rho}$, 


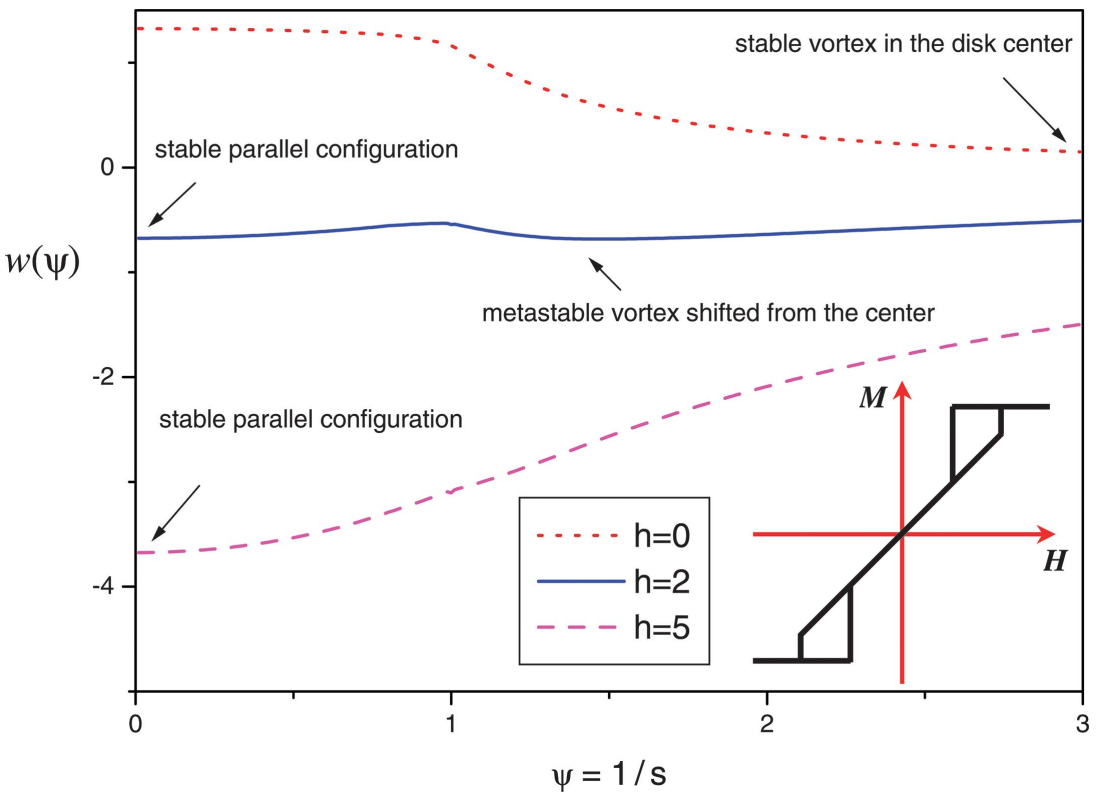

FIG. 4. (Color online) The evolution of the dependence of the total energy $w(\psi)$ when lowering the applied in-plane magnetic field $h$ $\equiv H / M_{s}$, for the parameters chosen at point A of the diagram in Fig. 3(a). For high $h$ (e.g., $\left.h=5\right)$ there is only one minimum of the energy $w$ corresponding to the parallel spin state (the bottom dashed line). For lower values of the applied field, a metastable energy minimum associated with a vortex inside the disk appears and then deepens when further lowering $h$ (the continuous line). Finally, for even lower values of $h$, the minimum energy corresponding to the parallel spin state disappears (the top dotted line) and the spin arrangement discontinuously changes to a vortex state. The corresponding magnetization loop is shown in the right bottom corner.

$m_{\phi}$, and $m_{z}$ are the magnetization components using a cylindrical coordinate system $(\rho, \phi, z)$ with its origin at the center of the rigid vortex [Fig. 1(a)]. Also $\vartheta$ denotes the angle between the local magnetization and the $z$-axis. The $z$-axis in Fig. 1 is perpendicular to the page and directed upwards (towards the reader).

As was done in Refs. 21 and 60, hereafter we will neglect the vortex core region. Note that as soon as the radius of the dot is larger than the vortex core, the contribution of the core region to the total energy gives a physically irrelevant energy offset when the magnetic vortex is inside the dot. Of course, this assumption becomes invalid and we, strictly speaking, should take the core into account when the vortex approaches the dot side surface from both inside and outside of the dot. However, the applicability of the "rigid" vortex model itself becomes problematic in this case due to the elliptical deformation of the vortex spin arrangement (see, for instance, Ref. 22). Thus, we can neglect the vortex core in the whole region of the applicability of the rigid vortex model. In such a case the magnetization can be approximated by $\boldsymbol{m}= \pm \boldsymbol{e}_{\phi}$ with the $\phi$-unit vector $\boldsymbol{e}_{\phi}$.

In this case the total dimensionless energy $w$ (normalized by $\left.M_{s}^{2} \pi R^{2} L\right)$ depends on the exchange length $R_{0}=\sqrt{C / M_{s}^{2}}$, the disk radius $R$, the dimensionless in-plane magnetic field $h=H / M_{s}$, and the aspect ratio $\beta=L / R$ with the dot thickness $L$. Considering contributions from the exchange $w_{\mathrm{ex}}$, magnetostatic $w_{m}$, and Zeeman $w_{h}$ energies (see, Appendix A) we derive

$$
\begin{aligned}
w(s)= & -\frac{R_{0}^{2}}{2 R^{2}}\left[\ln \left(1-s^{-2}\right)-A\right]-\frac{4 h \cos \varphi}{3 \pi} G(s)+w_{m}, \\
& s>1+b,
\end{aligned}
$$

$$
w(s)=\frac{R_{0}^{2}}{2 R^{2}} \ln \left(1-s^{2}\right)-\frac{4 h \cos \varphi}{3 \pi} s G\left(s^{-1}\right)+w_{m}, \quad 1-s>b,
$$

with magnetostatic energy

$$
\begin{aligned}
w_{m}= & \frac{s^{2}}{2 \pi} \int_{-\pi}^{\pi} d \phi \int_{-\pi}^{\pi} d \phi^{\prime} \\
& \times \frac{K\left(\phi-\phi^{\prime}, \beta\right) \sin \phi \sin \phi^{\prime}}{\sqrt{1+s^{2}-2 s \cos \phi} \sqrt{1+s^{2}-2 s \cos \phi^{\prime}}},
\end{aligned}
$$

and

$$
\begin{aligned}
\mathcal{K}\left(\phi-\phi^{\prime}, \beta\right)= & \ln \frac{\sqrt{2\left[1-\cos \left(\phi-\phi^{\prime}\right)\right]+\beta^{2}}+\beta}{\sqrt{2\left[1-\cos \left(\phi-\phi^{\prime}\right)\right]+\beta^{2}}-\beta} \\
& -\frac{2}{\beta}\left[\sqrt{2\left[1-\cos \left(\phi-\phi^{\prime}\right)\right]+\beta^{2}}\right. \\
& \left.-\sqrt{2\left[1-\cos \left(\phi-\phi^{\prime}\right)\right]}\right] .
\end{aligned}
$$

The function $G(s)$ can be expressed as

$$
G(s)=\left[\left(s^{2}+1\right) E\left(s^{-1}\right)-\left(s^{2}-1\right) K\left(s^{-1}\right)\right]
$$

using the complete elliptic integrals of the first and second kinds $K$ and $E$, respectively. The constant

$$
A=\ln \left[\frac{b^{2}\left(4-b^{2}\right)}{(b+1)^{2}}\right]
$$

has been chosen to keep the continuity of the energy at $s$ $=1$. Here, the dimensionless displacement $s$ of the magnetic vortex from the center of the disk (normalized by $R$ ) and the 


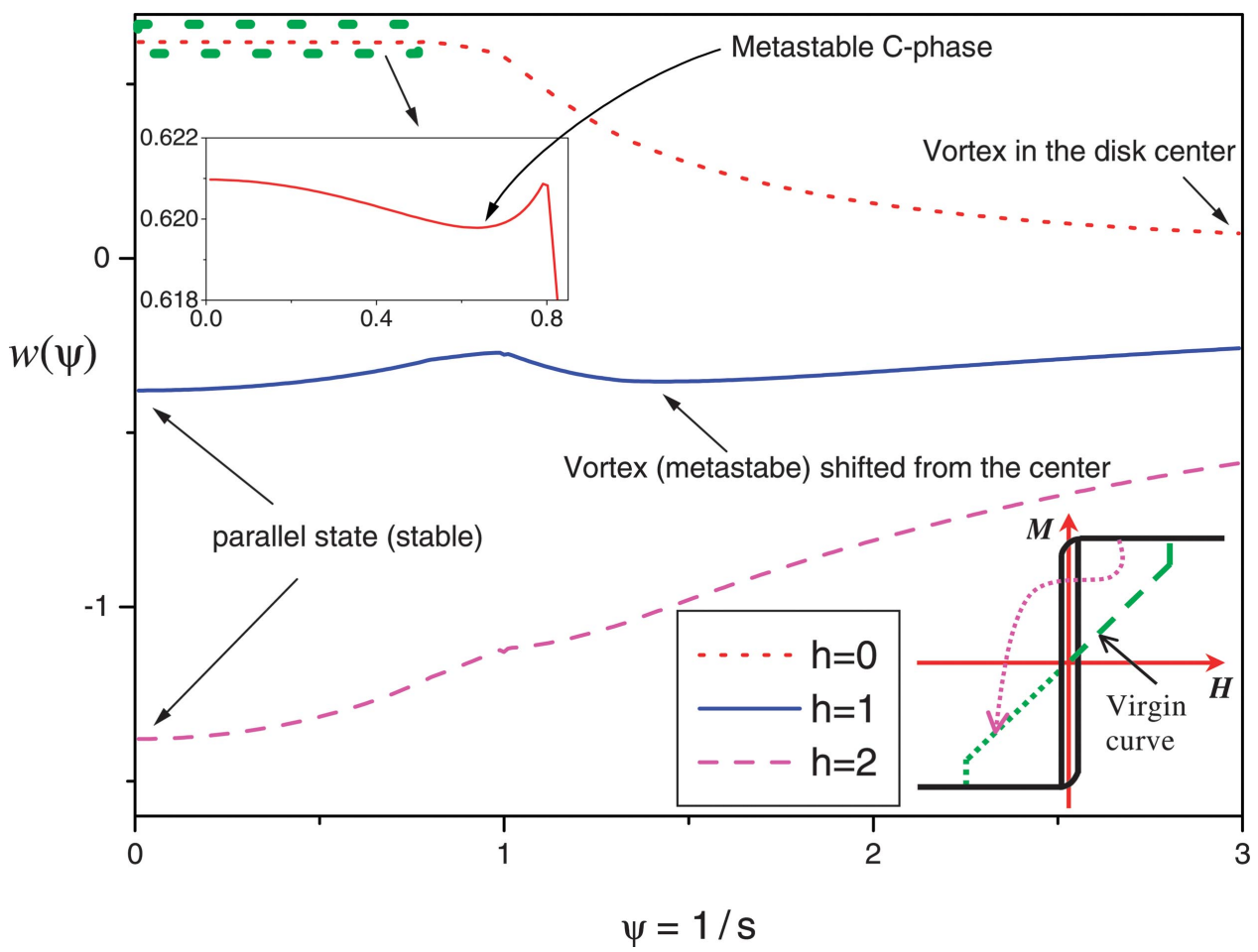

FIG. 5. (Color online) Total magnetic energy $w$ versus order parameter $\psi$ when lowering the in-plane magnetic field $h$ for the parameters chosen at point B on the diagram in Fig. 3(a). For relatively high magnetic fields, the observed change of $w(\psi)$ is similar to the one shown in Fig. 4 (the dashed curve at the bottom, $h=2$, and the middle continuous curve, $h=1$ ). However, the minimum in $w(\psi)$ ), originally corresponding to the parallel spin state, $\psi=0$, starts to shift to the right resulting in a second-order phase transition to the C-phase. In this case, the vortex begins to "continuously penetrate" the disk from infinity (see the left portion of the top dotted curve which is inside the left inset). The metastable C-phase $(\psi \neq 0)$ shown there continuously evolves from the parallel state at $\psi=0$. The minimum in $w(\psi)$ corresponding to the C-phase survives even at $h=0$. For very low negative fields, the C-phase rotates as a whole (Goldstone mode corresponding to a zero-energy rotation of $\boldsymbol{M}$ ) and the vortex state does not contribute to the reversal magnetization process even though it has a minimum energy at low fields. The corresponding magnetization loop with the virgin magnetization curve related to the vortex state is shown in the right bottom corner. The dashed arrow shows how one could access the vortex state by a sudden drop (or jump) of the external magnetic field.

dimensionless vortex core size $b=\tilde{b} / R$ have been introduced. The position of the magnetic vortex is also determined by the angle $\varphi$ in the polar coordinate system with its origin at the center of the dot [see Fig. 1(b)].

Expression (6) allows us (i) to analytically obtain the expansion of the total energy $w$ with respect to $\psi=1 / s$ (used below as an order parameter) in order to study phase transition (Fig. 3) when changing the applied magnetic field and (ii) to numerically calculate $w(1 / s)$ for any value of $1 / s$ (shown in Figs. 4-6).

\section{MAGNETIC ENERGY IN TERMS OF THE INVERSE VORTEX DISTANCE}

The usual Landau theory uses the magnetization as the order parameter to describe phase transitions in bulk magnetic materials (see Table I). However, an unusual type of spin arrangement-magnetic vortex — can be realized in small magnetic dots. This motivates us to introduce a novel order parameter for describing the evolution of magnetic phases when the external magnetic field changes.

In order to investigate the magnetization reversal process, we begin our considerations by first studying very high posi- tive in-plane magnetic fields. From the total energy $w$ in Eq. (6), it is clear that the minimum of the total energy $w(s)$ corresponds to $\varphi=0, s=\infty$. In other words, when the distance between the center of the disk and the center of the vortex is infinite then the energy is minimum for high magnetic fields. This is consistent with physical intuition. If we introduce the inverse vortex distance as

$$
\psi=\frac{1}{s},
$$

then $\psi$ is equal to zero for this parallel-spin phase. When the external in-plane magnetic field $\boldsymbol{H}$ decreases, this configuration can become unstable and a vortex can appear either inside or outside the dot. For the $\mathrm{C}$ phase and the vortex phase, the inverse vortex distance $\psi$ is nonzero, suggesting that $\psi$ could be chosen as an order parameter.

Let us write the expansion of the total energy $w$, in Eq. (6), with respect to $\psi$,

$$
w(\psi)=w(\psi=0)+A(h) \psi^{2}+B(h) \psi^{4}+\mathcal{O}\left(\psi^{6}\right) .
$$

If $\psi$ is small enough, the expansion can be truncated, keeping the first two $\psi$-dependent terms. Here we introduce two co- 


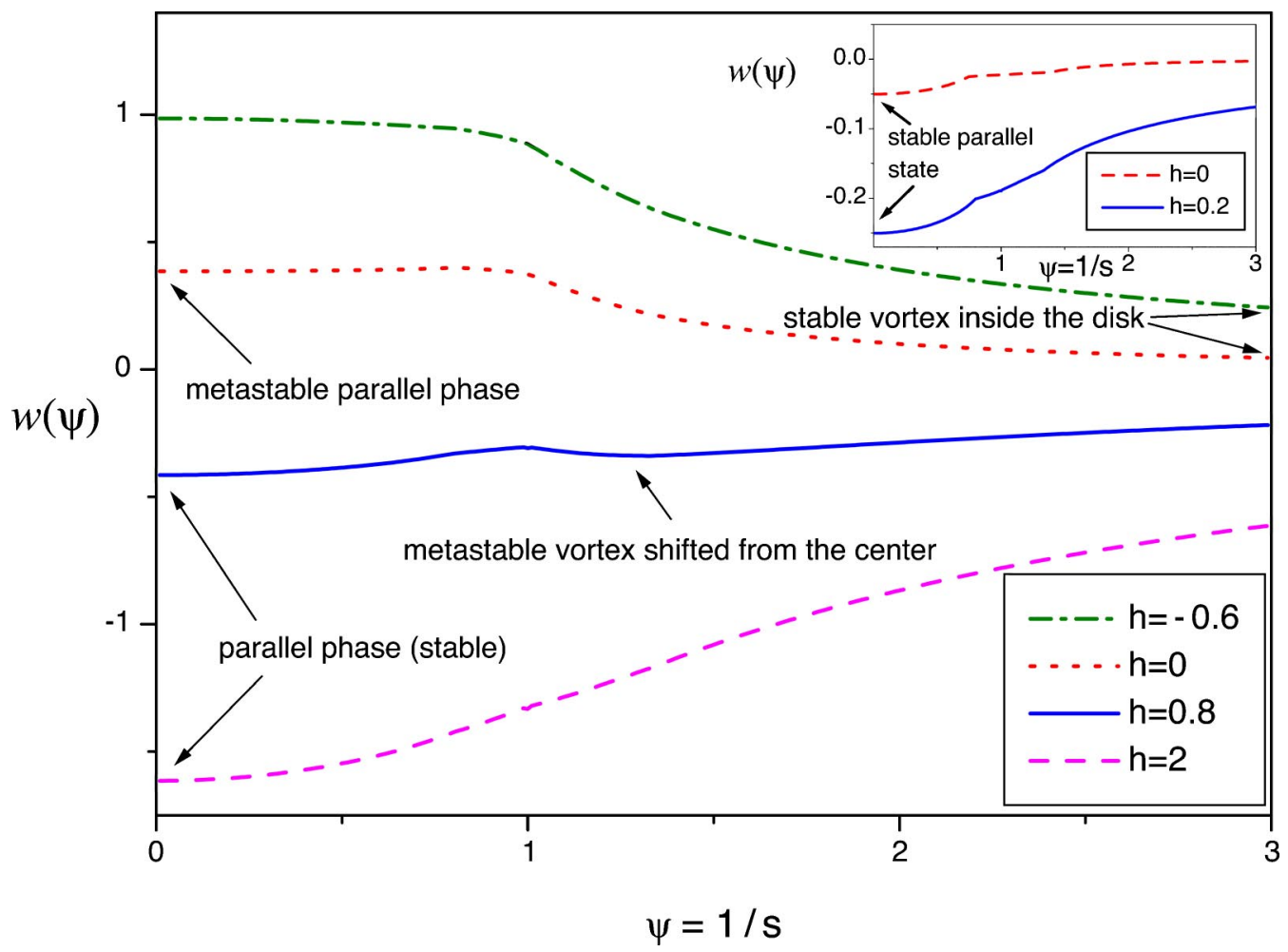

FIG. 6. (Color online) Main panel: dependence of the total energy $w(\psi)$ when lowering the in-plane magnetic field $h$ for the parameters chosen at point $\mathrm{C}$ on the diagram in Fig. 3(a). For high and positive $h$ (e.g., $h=2,0.8$ ), the behavior of $w(\psi)$ is similar to the one seen in Figs. 4 and 5 (the bottom dashed and the continuous curve). However, the parallel spin state is metastable even at zero magnetic field (the dotted line). For negative values of the external field, the parallel-spin state rotates as a whole (Goldstone mode). If the magnetic field suddenly drops to the negative value $h=-0.6$, then a gradient of the energy towards the vortex state appears (the top dotted-dashed curve). As shown in the top right inset, there is no energy minimum corresponding to the vortex state for the parameters corresponding to point D in Fig. 3(a).

efficients, $A(h)$ and $B(h)$ which resemble the ones used for standard Landau-type energy expansions. In the frame of the standard Landau approach these coefficients are chosen phenomenologically such as to obtain a second order phase transition (e.g., see Table I: $a$ has to change its sign and $b>0$ at a critical point) and satisfy the symmetry restrictions. In contrast to the phenomenological dependence of $a$ and $b$ on the system parameters, we have obtained a well-defined dependence of $A$ and $B$ on the dot radius, aspect ratio and magnetic field (see Appendix A for details):

$$
\begin{aligned}
& A(h)=w_{m}^{(2)}(\beta)+\frac{h}{8}+\frac{R_{0}^{2}}{2 R^{2}}, \\
& B(h)=w_{m}^{(4)}(\beta)+\frac{h}{64}+\frac{R_{0}^{2}}{4 R^{2}},
\end{aligned}
$$

where

$$
\begin{aligned}
w_{m}^{(2)}= & \frac{1}{2 \pi} \int_{-\pi}^{\pi} d \phi \int_{-\pi}^{\pi} d \phi^{\prime} \mathcal{K}\left(\phi-\phi^{\prime}, \beta\right) \sin \phi \\
& \times \sin \phi^{\prime}\left(\frac{1}{2}+\frac{3}{2} \cos 2 \phi+\cos \phi \cos \phi^{\prime}\right),
\end{aligned}
$$

$$
\begin{aligned}
w_{m}^{(4)}= & \frac{1}{2 \pi} \int_{-\pi}^{\pi} d \phi \int_{-\pi}^{\pi} d \phi^{\prime} \mathcal{K}\left(\phi-\phi^{\prime}, \beta\right) \sin \phi \sin \phi^{\prime} \\
& \times\left(\frac{11}{32}+\cos 2 \phi+\frac{35}{32} \cos 4 \phi+\frac{3}{4} \cos \phi \cos \phi^{\prime}\right. \\
& \left.+\frac{5}{4} \cos 3 \phi \cos \phi^{\prime}+\frac{9}{16} \cos 2 \phi \cos 2 \phi^{\prime}\right) .
\end{aligned}
$$

Note that here the coefficients $A$ and $B$ are driven by the magnetic field $h$, not the temperature, as in the usual Landau theory. Thus, changing the in-plane magnetic field $h$ can induce phase transitions. Several very substantial differences between the modified Landau-type approach derived here and the usual Landau theory for bulk magnets are summarized in Table I. The next section will consider phase transitions in the framework of the Landau energy obtained via a power-series expansion of $w(\psi)$.

\section{PHASE TRANSITIONS WHEN CHANGING THE MAGNETIC FIELD}

\section{A. $A$ and $B$ are positive in the Landau energy for any $h \geqslant 0$}

For high $h$, both $A(h)$ and $B(h)$ are positive for any values of the aspect ratio $\beta=L / R$ and reduced radius $R / R_{0}$. This 
corresponds to the minimum of $w(\psi)$ at $\psi=1 / s=0$, i.e., the parallel-spin state: the vortex is infinitely away from the center, and all spins are parallel to the external field. There is a region of parameters in the parameter space (aspect ratio, [reduced radius] $\left.]^{2}\right)=\left(\beta, R^{2} / R_{0}^{2}\right)$ [see Fig. 3(a), below the dashed line] where $A$ is positive even at $h=0$. For such parameters, the parallel spin state remains stable or meta-stable with respect to the vortex penetration even at zero field. The condition $A(h=0)=0$ determines the boundary [dashed line in Fig. 3(a)] of this parameter domain, corresponding to

$$
\frac{R^{2}}{R_{0}^{2}}=-\frac{1}{2 w_{m}^{(2)}(\beta)} .
$$

When the magnetic field changes sign, the system becomes unstable (see Appendix A) with respect to a rotation (around the dot center) of a vortex located far away from the center (essentially at infinity). In other words, the minimum at $\varphi=0$ becomes maximum as soon as $h$ changes sign and the angle $\varphi$ starts to increase (or decrease) with time until the system settles in its minimum when $\varphi= \pm \pi$. Thus, the magnetization reversal process proceeds via a rotation of the parallel spin state as a whole (Fig. 3(b)). Interestingly, the vortex in the center of the disk is stable (or metastable) in $h=0$ for the parameter domain

$$
\frac{R^{2}}{R_{0}^{2}}>\frac{1}{2 w_{m}^{(0)}}
$$

[above the continuous curve at the bottom of Fig. 3(a)] with

$$
w_{m}^{(0)}=\frac{1}{2 \pi} \int_{-\pi}^{\pi} d \phi \int_{-\pi}^{\pi} d \phi^{\prime} \mathcal{K}\left(\phi-\phi^{\prime}, \beta\right) \sin \phi \sin \phi^{\prime} .
$$

Of course, this stable (or metastable) state is not necessarily occupied, in the sense that the system might be trapped in some others local energy minimum. The last result Eq. (16), can be easily verified (see Appendix B) by expanding $w$ with respect to $s$ around $s=0$ as done in Refs. 11 and 21. Therefore, in the region of parameters between the continuous and dashed curves in Fig. 3(a), the magnetic vortex can be either stable or metastable at low magnetic fields but the vortex state does not contribute to the magnetization reversal process. However, if the magnetic vortex is created in the center (for instance, via a thermal activation process at high enough temperatures) the initial magnetization (virgin) curve has to be observed. This virgin curve is not accessible any more during the steady magnetization reversal process. This is consistent with and elucidates several numerical results ${ }^{22-24}$ obtained earlier. Thus, the vortex state cannot be obtained during the magnetization reversal process because the system always follows the metastable minimum if the system is slowly driven. However, the system can be excited enough to reach the stable vortex state when the system is driven sufficiently fast. Only for a sufficiently small radius $R$ of the disk, no vortex can be nucleated inside the disk [region below the bottom continuous curve, which is far below the dashed curve in Fig. 3(a)].

\section{B. Second-order phase transition from parallel-spin to curved} spin state: $A\left(h_{c}\right)=0, \quad B\left(h_{c}\right)>0$

\section{Order parameter near critical points}

Next consider the region of parameters where $A(h=0)$ $<0$, i.e., above the dashed line in Fig. 3(a). In this case, the parallel spin configuration (or parallel spin state) becomes unstable at

$$
h_{c}=-8 w_{m}^{(2)}-\left(\frac{2 R_{0}}{R}\right)^{2} .
$$

In this subsection we consider the case when $B\left(h_{c}\right)>0$. This inequality can be rewritten as

$$
\frac{R^{2}}{R_{0}^{2}}<\frac{3}{2 w_{m}^{(2)}-16 w_{m}^{(4)}},
$$

corresponding to the parameter domain below the top dotted line in Fig. 3(a). If $B\left(h_{c}\right)>0$, then our energy is formally similar to the usual Landau functional for second-order phase transitions. Namely, when lowering $h$, a second order phase transition occurs from the parallel spin configuration to a curved spin configuration (i.e., the magnetic vortex sitting outside the disk, also known as the C-phase) at $h=h_{c}$ [Fig. 3(b)]. This happens in the parameter region between the top dotted and dashed lines in Fig. 3(a). In such a case, the magnetic vortex starts to continuously move from infinity towards the dot center when the external magnetic field decreases. In other words, the order parameter $\psi$ continuously increases from $\psi=0$ to

$$
\psi=\psi_{\mathrm{eq}}=\frac{1}{s_{\mathrm{eq}}}=\sqrt{-\frac{A}{2 B}}=\sqrt{\frac{h_{c}-h}{16 B}} .
$$

\section{Critical slowing down near the critical points}

Interestingly, the dynamical properties also change abruptly at the phase transition between the C-phase and the parallel-spin phase. For instance, small deviations $\delta \psi$ from the equilibrium state $\psi_{\text {eq }}$ can be described by the following equation $\eta d s / d t=-\partial w / \partial s$, according to the overdamped magnetic vortex dynamics with viscosity $\eta$. This equation for $s$ can be rewritten as

$$
\begin{gathered}
\frac{d(\delta \psi)}{d t}=-\frac{(\delta \psi)}{\tau}, \quad \text { for } h<h_{c}, \\
\frac{d \psi}{d t}=-\frac{2 A}{\eta} \psi^{5}, \quad \text { for } h>h_{c},
\end{gathered}
$$

where $\delta \psi=\psi-\psi_{\mathrm{eq}}$ is the deviation from the equilibrium solution $\psi_{\text {eq }}=\sqrt{A /(-2 B)}$, and

$$
\tau=-\frac{\eta B^{2}}{A^{3}}=\frac{512 \eta B^{2}}{\left(h_{c}-h\right)^{3}}
$$

for $h<h_{c}$. As a consequence, the deviation $\psi_{0}-\psi_{e q}$ with 


$$
\psi(t=0)=\psi_{0}=\frac{1}{s_{0}} \neq \psi_{\mathrm{eq}}=\frac{1}{s_{\mathrm{eq}}}
$$

from the equilibrium decays exponentially in the $\mathrm{C}$ phase:

$$
\delta \psi=\left(\psi_{0}-\psi_{\mathrm{eq}}\right) \exp \left(-\frac{t}{\tau}\right) ; \quad s-s_{\mathrm{eq}}=\left(s_{0}-s_{\mathrm{eq}}\right) \exp \left(-\frac{t}{\tau}\right)
$$

Equation (22) shows that when the magnetic field approaches $h_{c}$ from below $\left(h<h_{c}\right)$ the relaxation time $\tau(h) d i$ verges showing the so-called "critical slowing down" when approaching the critical point.

In contrast to the exponential decay for $h<h_{c}$, the magnetic vortex approaches the parallel-spin state when $h>h_{c}$ following the power law

$$
\psi(t)=\left[\frac{2 A t}{\eta}+\psi_{0}^{-4}\right]^{-1 / 4} ; \quad s=\left[\frac{8 A t}{\eta}+s_{0}^{4}\right]^{1 / 4} .
$$

Note that the relaxation also becomes slower then $h$ approaches $h_{c}$ from above, $h>h_{c}$, because $A \propto\left(h-h_{c}\right) \rightarrow 0$. Therefore, the dynamics of the spin system has to drastically change at this second-order phase transition.

Interestingly, the spin dynamics remains the same before and after the discontinuous transition (the rotation of the parallel-spin state) at $h=0$ for the case discussed above when $A(h)>0$ and $B(h)>0$ for any $h \geqslant 0$; the dynamics can only change at $h=0$ due to the switching between the two rotated parallel-spin states.

\section{Evolution of spin configurations at low fields: Transition between $C$ and vortex phases}

When the magnetic field decreases further, the C-phase can either survive until $h=0$ or transform to the vortex state [Fig. 3(b)]. In order to estimate the stability of the C-phase at $h=0$ we can use the criterion $\psi(h=0)<1$, which can be rewritten in the form,

$$
B(0)>\frac{h_{c}}{16}
$$

This approximately gives the boundary of stability of the $C$-phase at $h=0$, within the frame of the "rigid" vortex model for $R<R_{C}$ with

$$
\frac{R_{C}^{2}}{R_{0}^{2}}=-\frac{1}{2 w_{m}^{(4)}+w_{m}^{(2)}}
$$

This is shown in Fig. 3(a) between the dashed and continuous lines. Therefore, when $R<R_{C}$, the $\mathrm{C}$ phase exists down to $h=0$ and then at $h<0$ the system becomes unstable with respect to rotations of the vortex center around the dot center: the minimum at $\varphi=0$ becomes maximum and the system rotates to the new minimum at $\varphi= \pm \pi$.

If $R>R_{C}$ the C-phase becomes unstable with respect to the nucleation of a magnetic vortex inside the dot. One can use the criterion $\psi\left(h_{n}\right) \approx 1$ (condition when vortex crosses the dot surface, $s=1)$ to estimate the nucleation field $h_{n}$. Using this criterion we obtain the region of stability of the C-phase [Fig. 3(b)]; a quantitative comparison of these analytical calculations with micromagnetic simulations should be straightforward and could elucidate the limitations of the approach developed here. When the external magnetic field decreases further, $h<h_{n}$, the vortex center [Fig. 1(a)] evolves following the energy minimum of Eq. (6) for $s<1$ and reaches zero at $h=0$. When the magnetic field changes sign and increases in modulus the vortex is first displaced from the dot center [Fig. 3(b)] and then a phase transition to either the $\mathrm{C}$ or the parallel-spin phase occurs [Fig. 3(b)] when $s\left(-h_{\text {exit }}\right) \approx 1$ (see, e.g., Refs. 20 and 21 ). Because of the surface barrier, this transition has to be abrupt, namely firstorder.

\section{Transition between the parallel-spin and the vortex phase: $A\left(h_{c}\right)=0, \quad B\left(h_{c}\right)<0$}

Now consider the case when $B\left(h_{c}\right)<0$, i.e., the parameter region above the dotted line in Fig. 3(a). Interestingly, the usual argument, that the stability of the system requires $B$ $>0$, is not applicable to our analytically-derived energy expansion in (12). Indeed, it is important to stress that the stability is determined by the nonexpanded energy (6), and not the truncated one in (12). In other words, the coefficients at higher powers of $\psi$ (i.e., $\psi^{6}, \psi^{8}, \ldots$ ) are responsible for the system stability (see Table I).

For high magnetic fields $h>h_{c}$ [i.e., $A(h)>0, B(h)<0$ in Eq. (4)], there is a maximum of the energy $w(\psi)$ at $\psi_{\max }$ $=\sqrt{A /(-2 B)}$ and minimum at $\psi=0$ corresponding to the parallel-spin state. This maximum (at $\psi_{\max }$ ) approaches the minimum (at $\psi=0$ ) when decreasing the magnetic field. At $h=h_{c}$ the maximum reaches $\psi=0$, while the minimum disappears. As soon as the applied magnetic field $h$ becomes lower than $h_{c}$, there is no minimum nearby $\psi=0$ and the system abruptly (discontinuously) goes to the vortex state [Fig. 3(b)]. Thus, instead of a second-order phase transition, a first order phase transition occurs in the parameter region $B\left(h_{c}\right)$ $<0$ above the top dotted line in Fig. 3(a) (see also Table I). This corresponds to a microdisk with large radius $R$. Intuitively, large-radius disks can abruptly accommodate a vortex inside it from the large- $h$ straight parallel-spin phase. Mechanically, it corresponds to a sudden "curling" transition of a compressed rod (see Sec. VI A below). Furthermore, this sharp transition between the $C$ and vortex phases agrees well with the sharp magnetization drop obtained earlier by micromagnetic simulations (see, e.g., Ref. 16).

\section{EVOLUTION OF THE TOTAL ENERGY WITH MAGNETIC FIELD: NUMERICAL CALCULATIONS}

\section{A. Controlling the magnetic state of a dot by slowly changing the applied magnetic field}

To finalize our analysis in the frame of the "rigid vortex" model, we performed numerical calculations of the total energy $w$ in Eq. (6) when changing the magnetic field $h$. We chose the parameters, the reduced radius $R / R_{0}$ and the aspect 
ratio $\beta=L / R$, from domains with different stable and metastable states [at the points A, B, C, D indicated in Fig. 3(a)].

Figure 4 shows the evolution of the energy when $h$ decreases, for the parameters $R / R_{0}$ and $\beta$ corresponding to point A in Fig. 3(a). At high $h$ there is only one minimum of $w(\psi)$ corresponding to the parallel-spin state (the parallel spin configuration has a minimum in the dashed line at the bottom). At lower fields, a metastable energy minimum appears which is associated with the vortex state inside the disk (continuous line). This energy minimum deepens while the minimum at $\psi=0$ for the parallel spin configuration flattens. Finally, the minimum of the parallel spin configuration at $\psi=0$ disappears and the vortex abruptly enters the disk (top dotted curve in Fig. 4). The corresponding schematic magnetization loop is shown in the inset of Fig. 4.

For the set of parameters corresponding to point B in Fig. 3(a), the evolution of $w(\psi)$, when $h$ decreases from high values to zero, is shown in Fig. 5. The behavior of $w(\psi)$, with changing $h$, is similar to the previous case when $h$ $>h_{c}$. When lowering $h$ further, the energy minimum previously located at $\psi=0$ starts to continuously move towards higher values of $\psi$ (see the left-top inset in Fig. 5 which magnifies a segment of the dotted curve on top). This corresponds to a second-order phase transition from the parallel spin configuration (the vortex is located at infinity, $\psi=0$ ) to the $\mathrm{C}$-phase (where the vortex is at a finite distance outside the disk $\psi \neq 0$ ) [Fig. 3(b)]. For the case of parameters corresponding to point B in Fig. 3(a), the minimum of $w(\psi)$ associated with the C-state exists even at zero $h$ (see the lefttop inset in Fig. 5). When the magnetic field changes polarity this C-phase rotates around the center of the disk Fig. 3(b). This rotation costs zero additional energy and corresponds to a Goldstone mode moving at the bottom of a "mexican-hat" shaped potential. Note that such potentials appear in different fields of physics, including: quantum field theory and cosmology, ${ }^{61-63}$ critical phenomena, equilibrium and nonequilibrium thermodynamics, ${ }^{64}$ superconductivity, superfluidity, vortex dynamics ${ }^{65}$ in superconductors, and even the theory of polyatomic molecules. ${ }^{66}$ However, in the case considered here, the hat has an additional minimum at the center of the hat, like in many real hats. Therefore, for these parameters, the magnetic vortex state does not contribute to the magnetization reversal process, while it has a minimum energy at low magnetic fields in agreement to the recent micromagnetic simulations. ${ }^{22-24}$ The corresponding magnetization loop is shown in the right-bottom inset of Fig. 5.

The discussed Goldstone mode does not depend on the shortcomings of the considered model. Indeed, at zero magnetic fields, the magnetization of small dots is not necessarily zero and the Goldstone mode describes the rotation of the magnetization vector. In a real system, with magnetic anisotropy or an anisotropy related to an unperfect dot shape, this rotation can cost some energy. However, when the asymmetry is weak, one can treat this rotation of $\boldsymbol{M}(h=0)$ as a Goldstone mode.

The evolution of the total energy $w(\psi)$, when changing the magnetic field $h$ for the parameters corresponding to the point $\mathrm{C}$ in Fig. 3(a), is shown in Fig. 6. The minimum in $w(\psi)$, corresponding to the parallel spin state, $\psi=0$, survives even at $h=0$ (the local minimum in the dotted line in Fig. 6). When $h$ changes its sign, the parallel-spin state rotates as a whole [Fig. 3(b)] around the dot center (another Goldstone mode) even though the vortex configuration has a minimum energy. The situation corresponding to point $\mathrm{D}$ is shown in the inset of Fig. 6. For this case the magnetic vortex is unstable for any value of the magnetic field.

\section{B. Controlling the magnetic state of a dot via fast, nonequilibrium change in the applied magnetic field}

Now, let us again consider the region of parameters corresponding to points $\mathrm{B}$ and $\mathrm{C}$ in Fig. 3(a). The question arises: Is it possible or not to reach the vortex spin configurations having the minimum energy at low fields? One of the possibilities is via thermal activation at high enough temperatures. Another possibility is to reach the minimum energy state via fast jumps of the external field (an example is shown by the dotted arrow in the right-bottom inset in Fig. 5). In such a case the system is brought far from both the stable and metastable minima. Thus, the spin configuration is exposed to an attraction of these two energy minima: the basin of attraction in the energy landscape towards the vortex state and the separate basin corresponding to a rotation as a whole. These two basins of attraction compete. This can be seen in the energy profile having a gradient towards a minima for the vortex state in Fig. 6, the dotted-dashed line. Depending on which minima is closer (namely, which minima the system can approach quicker: the vortex state or the rotated state), the system can evolve towards the parallelspin state or towards a vortex state.

For instance, if the applied magnetic field suddenly changes (during a time scale which is much shorter than $\eta$ ) from a high positive field (say, $h_{1}=2$ ) to a certain negative value (say, $h_{2}=-0.6$ ) for the dot parameters used to plot the total energy $w$ in Fig. 6, then the magnetic vortex begins to move towards the dot center from infinity and simultaneously to rotate around the disk. In order to reach the boundary of the disk, the magnetic vortex needs a time

$$
t_{\infty \rightarrow \text { disk }} \sim \eta\left|\int_{1}^{\infty} \frac{d s}{\frac{\partial w}{\partial s}\left(s, \phi=0, h_{2}\right)}\right| .
$$

If this time $t_{\infty \rightarrow \mathrm{disk}}$ is shorter than the time

$$
t_{\text {rotation }} \sim \eta\left|\int_{0}^{\pi} \frac{d \phi}{\frac{\partial w}{\partial \phi}\left(s=1, \phi, h_{2}\right)}\right|,
$$

which the vortex needs to rotate around the disk, than the vortex state is settled in. Otherwise, the system is set in the parallel-spin state. Thus, changing the timing and the amplitude of the external magnetic field jumps we can better control the spin configurations in magnetic dots. This will be further explored elsewhere. 
TABLE II. Comparison between our Landau theory approach to study mechanical buckling instabilities with the order parameter of another mechanical instability, known as the Portevin-Le Châtelier (PLC) effect or stick-slip plastic deformation (Ref. 28). The energy versus order parameter for the buckling case is considered here, but the analog for the PLC effect is not known yet. Thus, the five cells at the bottom right of the table, for the PLC effect, remain an open problem.

\begin{tabular}{|c|c|c|}
\hline & Euler buckling instability & Stick-slip plastic effect \\
\hline Order parameter & $\begin{array}{l}\qquad \psi_{b} \text { (a spatial } \\
\text { order parameter, the amplitude of } \\
\text { the destabilizing mode) }\end{array}$ & $\begin{array}{c}\psi_{p}=1 / \tau_{\text {bursts }} \text { (a temporal } \\
\text { order parameter, the number of } \\
\text { bursts per unit time) }\end{array}$ \\
\hline $\begin{array}{l}\text { Driving parameter } \\
\text { corresponding to } T \\
\text { in the usual approach }\end{array}$ & $\begin{array}{c}\tilde{T}=1 / f \\
f \text { is the applied force } \\
\left|\widetilde{T}-\widetilde{T}_{c}\right| / \widetilde{T}_{c} \text { acts as a reduced temp. }\end{array}$ & $\begin{array}{l}\text { the relative deformation } \epsilon \text {, } \\
\qquad\left|\epsilon-\epsilon_{c}\right| \text { acts as } \\
\text { the reduced temperature }\end{array}$ \\
\hline $\begin{array}{l}\text { Low force } f<f_{c} \\
\text { (high } \tilde{T}>\widetilde{T}_{c} \text { ) } \\
\text { Low relative deformation }\end{array}$ & $\begin{array}{c}\psi_{b}=0 \\
\text { straight bar }\end{array}$ & $\begin{array}{c}\psi_{p}=1 / \tau_{\text {bursts }}=0 \\
\text { compression with no plastic jump } \\
\text { deformations ("Laminar" flow phase) }\end{array}$ \\
\hline $\begin{array}{l}\text { High force } f>f_{c} \\
\text { (low } \tilde{T}<\tilde{T}_{c} \text { ) } \\
\text { High relative deformation }\end{array}$ & $\begin{array}{c}\psi_{b} \neq 0, \\
\text { curved (bent) bar }\end{array}$ & $\begin{array}{l}\qquad \psi_{p}=1 / \tau_{\text {bursts }} \neq 0 \\
\text { intermittent plastic compression } \\
\text { with sudden jumps ("turbulent-like") }\end{array}$ \\
\hline Energy [order parameter] & $\begin{array}{l}F_{b}\left[\psi_{b}\right]=F_{b 0}+A_{b} \psi_{b}^{2} \\
\quad+B_{b} \psi_{b}^{4}+\mathcal{O}\left(\psi_{b}^{6}\right)\end{array}$ & \\
\hline $\begin{array}{l}\text { Odd powers are } \\
\text { zero because of }\end{array}$ & $\begin{array}{c}\text { (1) symmetry } \\
F_{b}\left[\psi_{b}\right]=F_{b}\left[-\psi_{b}\right]\end{array}$ & \\
\hline $\begin{array}{l}\text { Energy expansion } \\
\text { coefficients }\end{array}$ & $\begin{array}{l}\text { (2) it can also be derived } \\
\text { from theory of elasticity }\end{array}$ & \\
\hline Coefficient of $\psi_{b}^{2}$ & $A_{b} \propto\left(f_{c}-f\right)$ or $A_{b} \propto\left(\widetilde{T}-\widetilde{T}_{c}\right)$ & \\
\hline Coefficient of $\psi_{b}^{4}$ at $f=f_{c}$ & $\begin{array}{c}B_{b}\left(f_{c}\right)>0, \text { second-order } \\
\text { (continuous) transition; } \\
B_{b}\left(f_{c}\right)<0, \text { first-order } \\
\text { (discontinuous) transition }\end{array}$ & \\
\hline
\end{tabular}

\section{BUCKLING INSTABILITIES}

\section{A. First versus second order buckling phase transition}

Our approach to describe phase transitions in micromagnetic dots, including "magnetic buckling," has several deep physical analogies with both the elastic and plastic systems discussed in the introduction. Some of these are compared in Tables I and II. This section is devoted to present a modified Landau-type theory approach to describe buckling transitions in an inextensible rod embedded in an elastic medium. This system was studied using a conventional mechanics approach, as in Chap. II of Ref. 38. The problem discussed here could be applicable to the buckling of very small rods which strongly interact with their environment, as, for instance, for charged carbon nanotubes ${ }^{45}$ or carbon nanotubes embedded in an elastic medium.

For arbitrary strong deflections $y(l)$ (see Fig. 7) parametrized by the arclength $l\left(0 \leqslant l \leqslant l_{\max }\right)$, the energy functional $F_{b}$ of a rod having circular cross section can be written (see Appendix C) as

$$
F_{b}=\int_{0}^{l_{\max }} d l\left\{\frac{I E\left(y^{\prime \prime}\right)^{2}}{2\left(1-\left(y^{\prime}\right)^{2}\right)}+f\left(\sqrt{1-\left(y^{\prime}\right)^{2}}-1\right)+\frac{\alpha}{2} y^{2}\right\} .
$$

Here we introduce the elastic modulus $E$ and the moment of inertia $I$ of the rod, the mechanical force $f$ acting on the end of the rod in the longitudinal direction, and the elastic constant $\alpha$ of the external elastic medium. Hereafter, we use the notation $d / d l=^{\prime}$.

As an example, we consider a rod with hinged ends, i.e., $y(0)=y\left(l_{\max }\right)=y^{\prime \prime}(0)=y^{\prime \prime}\left(l_{\max }\right)=0$. In such a case the possible buckling modes are ${ }^{38}$

$$
y(l)=\psi_{b} \sin \left(\frac{n \pi l}{l_{\max }}\right)
$$

where we introduce the buckling order parameter $\psi_{b}$ and the undulation number $n$. Interestingly, the magnetic buckling shape $\left(\mathrm{C}, \mathrm{S}, \mathrm{W}\right.$, etc. $\left.{ }^{16}\right)$ and the vorticity in mesoscopic 


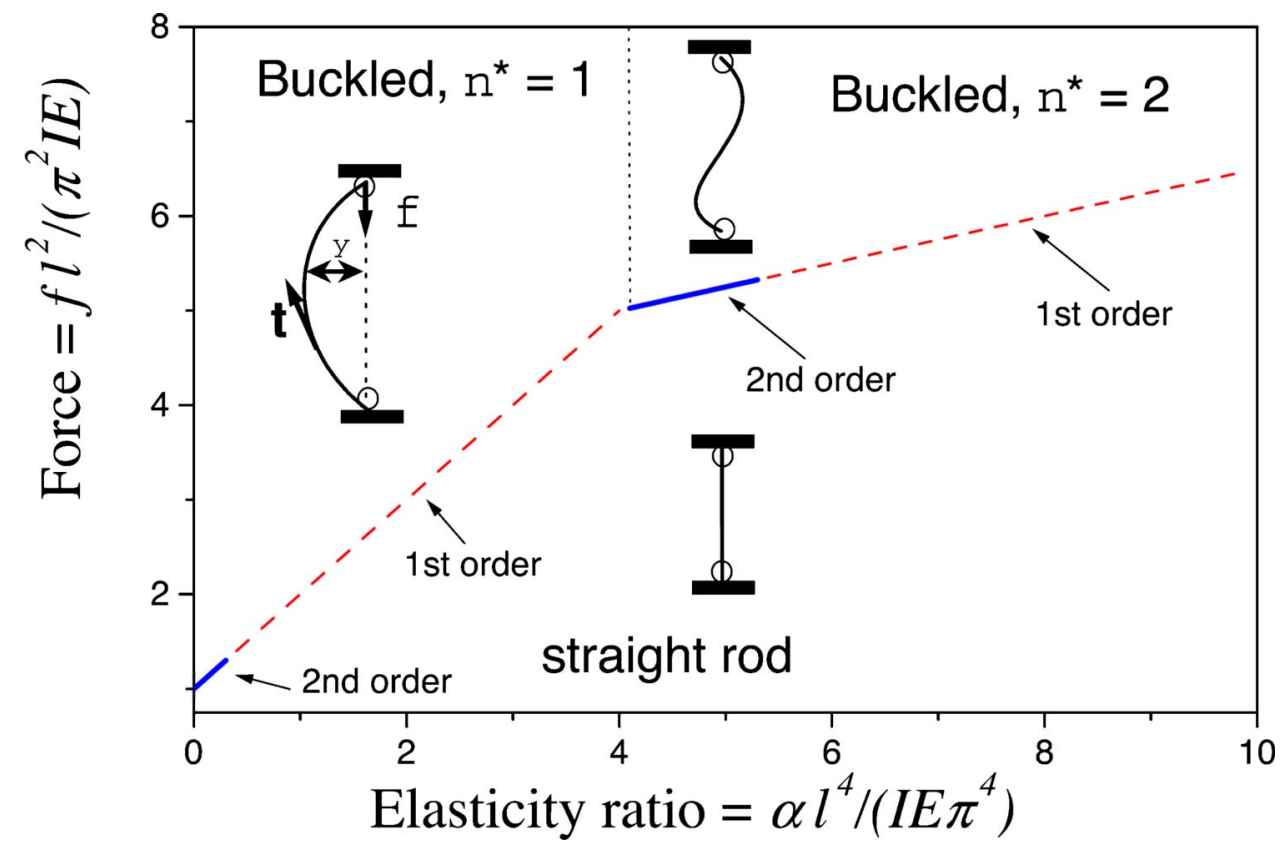

FIG. 7. (Color online) Phase diagram for the mechanical buckling instability. The parameters shown are the normalized force $=f l^{2} /\left(\pi^{2} I E\right)$ versus the ratio of the medium/rod elasticities $\alpha l^{4} /\left(\pi^{4} I E\right)$. Solid segments correspond to second-order phase transitions from the straight rod state to the buckled state, while the first-order phase transitions are indicated by the dashed lines. The different buckling modes, with different undulation numbers $n^{*}$, are sketched in the corresponding phase domains. The black vertical dotted segment around $\alpha l^{4} /\left(\pi^{4} I E\right)$ $\approx 4$ corresponds to a first-order transition between the $n^{*}=1$ (C-phase-like) and the $n^{*}=2$ (S-phase-like). The transition from the straight rod phase to the $n^{*}=1$ buckled phase is the mechanical analog of the magnetic phase transition between the straight and bent spin-configuration phases: this diagram corresponds to the one shown in Fig. 3(b). The transition to the $n^{*}=2$ buckled rod state is the mechanical analog of the magnetic transition to the $\mathrm{S}$ phase in microdisks.

superconductors ${ }^{67-73}$ could be mapped to the undulation number $n$. Also charged rods, ${ }^{45}$ as well as the elastic blockade and Coulomb blockade of charged disks can be studied with this approach.

Substituting these buckling modes $y(l)$ into the energy functional, Eq. (30), and expanding $F_{b}\left(\psi_{b}\right)$ up to $\psi_{b}^{4}$, we get the Landau-type energy expansion

$$
F_{b}=A_{b}(f) \psi_{b}^{2}+B_{b}(f) \psi_{b}^{4}+\mathcal{O}\left(\psi_{b}^{6}\right)
$$

with

$$
\begin{gathered}
A_{b}=\frac{\pi^{2} n^{2}}{4 l_{\max }}\left(f_{c}(n)-f\right), \\
B_{b}=\frac{\pi^{4} n^{4}}{64 l^{3}}\left(\frac{4 I E n^{2} \pi^{2}}{l_{\max }^{2}}-3 f\right),
\end{gathered}
$$

and

$$
f_{c}(n)=\frac{I E \pi^{2}}{l_{\max }^{2}}\left(n^{2}+\frac{\alpha l_{\max }^{4}}{I E \pi^{4} n^{2}}\right) .
$$

Obviously, the transition from the straight rod to a buckled state occurs as soon as the external force exceeds the minimum value of the critical force $f_{c}(n)\left[f_{c}(n)\right.$ is consistent with Chap. II of Ref. 38, while $A_{b}$ and $B_{b}$ are new]. At this force, $f_{c}^{*}=f_{c}\left(n^{*}\right)=\min \left(f_{c}(n)\right)$, the rod becomes unstable with respect to the buckling mode with $n=n^{*}$ and a mechanical buckling phase transition occurs.
Our approach goes beyond the usual purely mechanical treatment and can predict the regime of parameters where the transition is either first or second order. Our approach also provides quantitative predictions for the buckling dynamics near critical points. As was discussed in previous sections, this buckling transition is of second order if $B_{b}\left(f=f_{c}^{*}\right)$ is positive. Otherwise, when $B_{b}\left(f=f_{c}^{*}\right)<0$, there is no energy minimum nearby $\psi_{b}=0$, at $f=f_{c}^{*}+0$, and the system discontinuously (via a first-order phase transition) changes to a strongly bent state. This is an analog of the magnetic vortex state in microdisks. The sign of $B_{b}\left(f_{c}^{*}\right)$ can be derived from the equation

$$
B_{b}\left(f_{c}^{*}\right)=\frac{\pi^{6}\left(n^{*}\right)^{4} I E}{64 l^{5}}\left(\left(n^{*}\right)^{2}-3 \frac{\alpha l^{4}}{I E \pi^{4}\left(n^{*}\right)^{2}}\right) .
$$

After minimizing $f_{c}(n)$ with respect to $n$, we can obtain the value of $B_{b}\left(f_{c}^{*}\right)$. From these results, we can construct the buckling phase diagram shown in Fig. 7. Note that this diagram corresponds to the one shown in Fig. 3(b) for magnetic microdisks.

More detailed analysis, including the transition between different metastable configurations as well as possible transition between buckling modes with different undulation number, will be presented elsewhere.

\section{B. Critical slowing down for a second-order buckling phase transition}

Now we consider the region of parameters (see Fig. 7) where the second order phase transition occurs. This covers 
the important case when there is no external elastic medium $(\alpha=0)$. When the externally applied force exceeds a critical threshold value $f_{c}^{*}=f_{c}\left(n^{*}\right)$, the buckling order parameter (or curvature of the rod) begins to increase continuously and follows the equation

$$
\psi_{b}(f)=\psi_{b}^{\mathrm{eq}}=\frac{\pi n^{*}}{2} \sqrt{\frac{f-f_{c}^{*}}{2 l_{\max } B\left(f_{c}^{*}\right)}}
$$

near the critical force $f_{c}^{*}$.

Next, we consider of how small deviations, from the equilibrium value $\psi_{b}^{\text {eq }}$, of the order parameter decay. The dynamical equation for the rod can be written as

$$
m_{\text {eff }} \ddot{\psi}_{b}+\eta \dot{\psi}_{b}=-\frac{d F_{b}}{d \psi_{b}}=-2 A_{b} \psi_{b}+4 B_{b} \psi_{b}^{3},
$$

where $m_{\text {eff }}=m / 2$ is the effective mass of the order parameter with the rod mass $m$, while $d / d t=^{\circ}$. The damping coefficient $\eta$ is determined by the energy dissipation during rod motion. For small deviations $\delta \psi_{b} / \psi_{b}^{\mathrm{eq}}$, with $\delta \psi_{b}=\psi_{b}-\psi_{b}^{\mathrm{eq}}$, we obtain

$$
\frac{m_{\mathrm{eff}}}{\eta}\left(\delta \ddot{\psi}_{b}\right)+\left(\delta \dot{\psi}_{b}\right)=-\frac{\left(\delta \psi_{b}\right)}{\tau_{b}}
$$

with relaxation time

$$
\begin{gathered}
\tau_{b}^{-1}=\frac{\pi^{2}\left(n^{*}\right)^{2}}{2 \eta l_{\max }}\left(f-f_{c}\left(n^{*}\right)\right) \text { for } f<f_{c}^{*}, \\
\tau_{b}^{-1}=\frac{\pi^{2}\left(n^{*}\right)^{2}}{\eta l_{\max }}\left(f_{c}\left(n^{*}\right)-f\right) \quad \text { for } \quad f>f_{c}^{*} .
\end{gathered}
$$

Therefore, the relaxation time $\tau_{b}(f)$ diverges at the phase transition $f_{c}^{*}$ and takes different values for different undulation numbers $n^{*}$ that minimize $f_{c}(n)$.

According to the dynamical equation (39), the buckling order parameter $\psi_{b}$ (or curvature of the rod) approaches the equilibrium showing several oscillations if

$$
\tau_{b}<\frac{4 m_{\mathrm{eff}}}{\eta} .
$$

However, the oscillations vanish when the mechanical force $f$ becomes closer to its critical value $f_{c}^{*}$. Very near to the critical point, the buckling order parameter decays exponentially

$$
\psi_{b} \propto \exp \left(-t / \tau_{b}\right) .
$$

Therefore, we predict that the compressed rod exhibits critical slowing down near the critical force $f_{c}^{*}$.

\section{CONCLUSIONS}

\section{A. Open problems}

Answering several important issues and presenting several physical analogies, this work can pose some questions to guide several future studies in both micromagnetic and nanomechanics:

(1) How to develop a more general theoretical framework related to the rigid vortex model for an array of interacting micromagnetic dots.

(2) Detailed micromagnetic simulations and experiments could be useful for further confirmation of the effects predicted here and for extending the models and ideas presented in this work.

(3) The generalization of our approach to the case when quantum effects are important, including a quantum treatment of the Goldstone modes studied here.

(4) When the applied magnetic field is perpendicular to the micromagnetic dot, there is a phase transition between the parallel-spin (for larger $H_{\perp}$ ) and a vortex phase (for smaller $H_{\perp}$ ). This transition can be easily described with a modified version of our approach.

(5) How to best study driven nonequilibrium phase transitions like the Portevin-Le Châtelier effect, and complete the five cells at bottom of Table II [e.g., what is the analog of the free energy for the Portevin-Le Châtelier (PLC) effect? Can we consider the PLC effect as a generalized cascade of buckling transitions?].

(6) Table II and Fig. 2 suggest a plethora of open problems involving the development of a generalized thermodynamics of the buckling instability. For instance, the analog of the free energy $F=U-T S$ would become

$$
F=(\text { Elastic energy })-(1 / f) S_{\text {bar }},
$$

where $S_{\text {bar }}$ would be the "effective entropy" of the bar, and $f$ is the externally applied longitudinal force acting (as an inverse temperature) on the elastic bar.

(7) Further investigations of illuminating and insightful analogies between phase transitions in micromagnetic dots, compressed rods, and nanosuperconductors. ${ }^{67-73}$

\section{B. Summary}

In conclusion, using the rigid magnetic vortex model, we studied phase transitions in the spin configuration of a microdisk when changing the external magnetic field. We analytically showed that five different sequences of phase transitions can be realized among three different spinconfiguration phases: parallel-spin phase, curved spin phase, and vortex phase. Which sequence is realized depends on the dot radius, $R$, aspect ratio $\beta=L / R$, and the exchange length $R_{0}$. The numbers (1), (2), (3), (4), (5) below refer to five different parameter regions in Fig. 3(a).

(1) The parallel-spin state transforms into the vortex state and vice versa via a first order phase transition.

(2) The C-phase mediates the transformation from the parallel-spin to the vortex state: a second-order phase transition occurs between the parallel-spin and the C-phase, while a discontinuous first-order transition occurs between the $\mathrm{C}$ phase and the vortex phase.

(3) The parallel-spin state can transform into a C-phase which rotates when the magnetic field changes sign. Even though the vortex phase has a minimum energy at low magnetic fields, it does not contribute to the magnetization reversal process and appears only during the initial magnetization. This rotation costs zero additional energy and corresponds to a Goldstone mode moving at the bottom of a "hat-shaped" potential. 
(4) The parallel-spin state rotates when the magnetic field changes sign. Neither the C-phase nor the vortex phase contribute to the magnetization reversal process here, although the vortex state has a minimum energy at low magnetic fields resulting in the initial magnetization curve. Note that in (3) the C-phase rotates when $h$ changes sign, while in (4) it is the straight parallel-spin phase that rotates.

(5) Only the parallel-spin state has an energy minimum. The $\mathrm{C}$ phase and the vortex phase cannot appear because they are completely unstable in this region.

The dynamical response of the order parameter can drastically change at the phase transitions, producing "critical slowing down" at the second order phase transition between the parallel-spin and the $\mathrm{C}$ phase. We want to stress that the physical scenarios obtained here are related to the spontaneous symmetry breaking at the transition between the parallelspin state and the bending or vortex states and, therefore, these results are more general than the rigid vortex model itself. Hence, this physical picture could be generalized to much more complicated spin configurations in circular thin dots, ${ }^{16,22,23}$ and can be used as a guide to look for novel ways to control the spin configuration in magnetic dots. We predict a "critical slowing down" at the second-order phase transition between the parallel-spin and the $\mathrm{C}$ phase. Recent micromagnetic simulations ${ }^{59}$ support our predicted "critical slowing down", thus, partly verifying the whole physical picture proposed here. Other predictions of our work are consistent with earlier micromagnetic computations. ${ }^{16,23,24}$

We have found that the buckling instability is a good mechanical analog of the magnetic buckling transition from the parallel-spin to a bent spin configuration (either C-phase or vortex phase). Using a substantially modified Landau theory for studying mechanical buckling instabilities of a compressed elastic rod embedded in an elastic medium, we prove that the transition to a buckled state can be either first or second order, depending on the ratio of the elasticity of the rod and the elasticity of the external medium. Also, critical slowing down was predicted for the second-order mechanical buckling transition.

\section{ACKNOWLEDGMENTS}

We acknowledge very stimulating discussions with V. Novosad and Y. Otani. This work was supported in part by the National Security Agency (NSA) and Advanced Research and Development Activity (ARDA) under Air Force Office of Research (AFOSR) Contract No. F49620-02-1-0334; and also supported by the U.S. National Science Foundation Grant No. EIA-0130383.

\section{APPENDIX A: TOTAL ENERGY OF THE RIGID MAGNETIC VORTEX AND ASYMPTOTICAL EXPANSION OF THE ENERGY}

Here we outline how to obtain the expression (6) for the total energy $w$ of a magnetic dot and how to derive the asymptotic expansion shown in Eq. (12).

In order to obtain the expressions for the exchange $w_{\mathrm{ex}}$ and magnetostatic $w_{m}$ energies, we use a coordinate system centered at the vortex core [see Fig. 1(a)]. The dimensionless exchange $w_{\text {ex }}$ and Zeeman $w_{h}$ energies can then be rewritten as

$$
\begin{aligned}
w_{\mathrm{ex}} & =\frac{R_{0}^{2}}{2 \pi R^{2}} \int_{\rho} \frac{1}{\rho} d \rho d \phi \\
& =\frac{R_{0}^{2}}{2 \pi R^{2}} \int_{-\phi_{m}}^{\phi_{m}} d \phi\left\{\ln \left[\rho_{1}(\phi)\right]-\ln \left[\rho_{2}(\phi)\right]\right\}, \\
w_{h} & =-\frac{h}{\pi R^{2}} \int \rho \cos \phi d \phi d \rho \\
& =-\frac{h}{2 \pi R^{2}} \int_{-\phi_{m}}^{\phi_{m}}\left\{\rho_{1}^{2}(\phi)-\rho_{2}^{2}(\phi)\right\} \cos \phi d \phi,
\end{aligned}
$$

where $\rho_{1,2}=R\left\{s \pm \sqrt{1-s^{2} \sin ^{2} \phi}\right\}$ [see Fig. 1(b)] and $\sin \phi_{m}$ $=1 / s$, if the center of the vortex is outside the dot; while $\rho_{1}=R\left\{\sqrt{1-s^{2} \sin ^{2} \phi}-s \cos \phi\right\}$ and $\phi_{m}=\pi$, if the magnetic vortex is inside the dot. Although $\rho_{2}=0$ when the magnetic vortex is inside the dot, the integration for the exchange energy $w_{\text {ex }}$ has to be cut off at a distance of about the vortex core size $b$. After an additional simple integration over $\phi$ we obtain the expression for the exchange and Zeeman energies presented in Eq. (6).

In order to derive the magnetostatic energy $w_{m}$, we can use the equation

$$
\sigma=\frac{M_{s} s \sin \phi}{\sqrt{1+s^{2}-2 s \cos \phi}}
$$

for the magnetic charge density $\sigma$ accumulated at the side surface of the dot. Here $\phi$ is now the angle in the cylindrical coordinate system with its origin in the dot center (Fig. 1(b)). Next, the distance between two elements on the side surface can be written as $\left|\boldsymbol{r}-\boldsymbol{r}^{\prime}\right|=\sqrt{2 R^{2}\left[1-\cos \left(\phi-\phi^{\prime}\right)\right]+\left(z-z^{\prime}\right)^{2}}$ where $\phi, \phi^{\prime}, z$, and $z^{\prime}$ are the coordinates of these surface elements. Therefore, the magnetostatic energy $w_{m}$ can be written as

$$
\begin{aligned}
w_{m}= & \frac{s^{2}}{2 L \pi} \int_{-\pi}^{\pi} \frac{\sin \phi \sin \phi^{\prime} d \phi d \phi^{\prime}}{\sqrt{1+s^{2}-2 s \cos \phi} \sqrt{1+s^{2}-2 s \cos \phi^{\prime}}} \\
& \times \int_{0}^{L} \frac{d z d z^{\prime}}{\sqrt{2 R^{2}\left[1-\cos \left(\phi-\phi^{\prime}\right)\right]+\left(z-z^{\prime}\right)^{2}}} .
\end{aligned}
$$

Integrating over $z$ and $z^{\prime}$, we derive the magnetostatic energy in Eq. (7).

Here it is important to stress that $\partial w / \partial \varphi=-\Gamma(s) h \sin \varphi$, and $\partial^{2} w / \partial \varphi^{2}=\Gamma(s) h \cos \varphi$, with $\Gamma(s)>0$ for any $s$. Thus, the minimum and the maximum of the total energy $w$ with respect to $\varphi$ occur at

$$
\varphi_{\min }=\frac{1-h /|h|}{2} \pi+2 \pi \tilde{n}, \quad \varphi_{\max }=\pi+\varphi_{\min }
$$

with integer $\tilde{n}$.

Now let us expand the total energy $w$ of a magnetic dot for large vortex displacements $s \gg 1$ and $\varphi=0$; i.e., we construct the asymptotic expansion with respect to $1 / s$ up to $1 / s^{4}$. For the exchange energy $w_{\text {ex }}$ we obtain 


$$
w_{\mathrm{ex}}=\left(\frac{R_{0}}{2 R}\right)^{2}\left[2 s^{-2}+s^{-4}+\mathcal{O}\left(s^{-6}\right)\right] .
$$

Expanding the complete elliptic integrals $K$ and $E$ in Eq. (9) we derive an expression for the Zeeman energy as

$$
w_{h}=-h+\left(\frac{h}{2^{3}}\right) s^{-2}+\left(\frac{h}{2^{6}}\right) s^{-4}+\mathcal{O}\left(s^{-6}\right) .
$$

After simple, but long, calculations one can derive an asymptotic expansion for the magnetostatic energy $w_{m}$, Eq. (7), as

$$
w_{m}=w_{m}^{(0)}+w_{m}^{(2)} s^{-2}+w_{m}^{(4)} s^{-4}+\mathcal{O}\left(s^{-6}\right) .
$$

Combining the asymptotic expansions for $w_{\mathrm{ex}}, w_{h}$, and $w_{m}$, and using the notation $\psi=1 / s$, we derive the modified Landau-type energy shown in Eq. (12).

\section{APPENDIX B: ENERGY EXPANSION FOR SMALL VORTEX DISPLACEMENTS FROM THE DOT CENTER}

The aim of this appendix is to derive an expansion of the total energy $w(h)$ in Eq. (6) for small values of $s$ and $\varphi=0$. Note that a similar expression was obtained earlier (see Refs. 20 and 21) and we just summarize a brief derivation of it only for the reader's convenience and for completeness. The exchange energy $w_{\mathrm{ex}}$ for small $s$ has the form:

$$
w_{\mathrm{ex}}=-\frac{R_{0}^{2}}{2 R^{2}} s^{2}+\mathcal{O}\left(s^{4}\right) .
$$

Hereafter, we omit an $s$-independent constant. The Zeeman energy $w_{h}$, up to second order with respect to $s$, is

$$
w_{h}=-h s+\mathcal{O}\left(s^{3}\right),
$$

while the magnetostatic energy $w_{m}$ can be expanded as

$$
w_{m}=w_{m}^{(0)} s^{2}+\mathcal{O}\left(s^{4}\right) \text {. }
$$

Next, minimizing the total energy $w$ we obtain the equation for the equilibrium vortex position $s_{v}$ as follows $2\left[w_{m}^{(0)}\right.$ $\left.-R_{0}^{2} /\left(2 R^{2}\right)\right] s_{v}=h$. This equation can be used to calculate the susceptibility and to estimate the annihilation field $h_{\text {exit }}{ }^{21}$

$$
\left|h_{\text {exit }}\right|=2\left(w_{m}^{(0)}-\frac{R_{0}^{2}}{2 R^{2}}\right) .
$$

Note that the total energy $w=w_{\mathrm{ex}}+w_{h}+w_{m}$ has a minimum at $s=s_{v}$, if $\partial^{2} w / \partial s^{2}\left(s=s_{v}\right)>0$. This condition becomes invalid when $w_{m}^{(0)}-R_{0}^{2} /\left(2 R^{2}\right)$ becomes negative. Therefore, there is no minimum corresponding to the vortex state if $R_{0}^{2} /\left(2 R^{2}\right)$ $>w_{m}^{(0)}$, as mentioned in the text.

\section{APPENDIX C: POTENTIAL ENERGY OF A LONGITUDINALLY COMPRESSED ROD}

Here we derive the expression for a longitudinally compressed inextensible rod embedded in an elastic medium. We start from the well known expression (see, e.g., Ref. 38) for the elastic energy of a rod having circular cross section with no torsion,

$$
F_{\text {elastic }}=\frac{I E}{2} \int_{0}^{l_{\max }} d l\left\{t \times \frac{d t}{d l}\right\}^{2},
$$

where $t$ is a unit vector tangential to the rod, $E$ is the elastic modulus, and $I$ is the moment of inertia of the rod. The mechanical force $f$ acting on the rod in the longitudinal direction provides an additional contribution $F_{f}$ to the energy of the curved rod,

$$
F_{f}=-f \int_{0}^{l_{\max }}\left(\frac{d x}{d l}-1\right) d l
$$

Hereafter we will use the coordinates of the bent rod: $x(l)$ and $y(l)$. These coordinates obey the equation: $d x^{2}+d y^{2}$ $=d l^{2}$. This can be rewritten as

$$
\frac{d x}{d l}=\sqrt{1-\left(\frac{d y}{d l}\right)^{2}} .
$$

The unit tangential vector and its derivative can be expressed via deflection as

$$
\begin{gathered}
t=\left(\frac{d x}{d l}, \frac{d y}{d l}\right)=\left[\sqrt{1-\left(\frac{d y}{d l}\right)^{2}}, \frac{d y}{d l}\right] ; \\
\frac{d t}{d l}=\frac{d^{2} y}{d l^{2}}\left(-\frac{d y / d l}{\sqrt{1-(d y / d l)^{2}}}, 1\right) .
\end{gathered}
$$

Using Eqs. (C4), we obtain

$$
F_{\text {elastic }}+F_{f}=\int_{0}^{l_{\max }} d l\left\{\frac{I E\left(y^{\prime \prime}\right)^{2}}{2\left[1-\left(y^{\prime}\right)^{2}\right]}+f\left(\sqrt{1-\left(y^{\prime}\right)^{2}}-1\right)\right\} \text {. }
$$

This equation was independently obtained in, e.g., Ref. 40. Finally, assuming an ideal elastic response of the external medium, with no anharmonic terms, we derive Eq. (30).
${ }^{1}$ R. P. Cowburn, D. K. Koltsov, A. O. Adeyeye, and M. E. Welland, Phys. Rev. Lett. 83, 1042 (1999).

${ }^{2}$ R. P. Cowburn and M. E. Welland, Science 287, 1466 (2000).

${ }^{3}$ T. Shinjo, T. Okuno, R. Hassdorf, K. Shigeto, and T. Ono, Science 289, 930 (2000).

${ }^{4}$ T. Pokhil, D. Song, and J. Nowak, J. Appl. Phys. 87, 6319
(2000).

${ }^{5}$ A. Fernandez and C. J. Cerjan, J. Appl. Phys. 87, 1395 (2000).

${ }^{6}$ M. Schneider, H. Hoffmann, and J. Zweck, Appl. Phys. Lett. 79, 3113 (2001).

${ }^{7}$ K. J. Kirk, S. McVitie, J. N. Chapman, and C. D. W. Wilkinson, J. Appl. Phys. 89, 7174 (2001). 
${ }^{8}$ A. Lebib, S. P. Li, M. Natali, and Y. Chen, J. Appl. Phys. 89, 3892 (2001).

${ }^{9}$ A. Wachowiak, J. Wiebe, M. Bode, O. Pietzsch, M. Morgenstern, and R. Wiesendanger, Science 298, 577 (2002).

${ }^{10}$ M. Schneider, H. Hoffmann, S. Otto, Th. Haug, and J. Zweck, J. Appl. Phys. 92, 1466 (2002).

${ }^{11}$ Y. Otani, H. Shima, K. Yu. Guslienko, V. Novosad, and K. Fukamichi, Phys. Status Solidi A 189, 521 (2002).

${ }^{12}$ V. Novosad, K. Yu. Guslienko, H. Shima, Y. Otani, S. G. Kim, K. Fukamichi, N. Kikuchi, O. Kitakami, and Y. Shimada, Phys. Rev. B 65, 060402(R) (2002).

${ }^{13}$ M. Natali, I. L. Prejbeanu, A. Lebib, L. D. Buda, K. Ounadjela, and Y. Chen, Phys. Rev. Lett. 88, 157203 (2002).

${ }^{14}$ X. Zhu, P. Grütter, V. Metlushko, and B. Ilic, Phys. Rev. B 66, 024423 (2002).

${ }^{15}$ V. Novosad, M. Grimsditch, J. Darrouzet, J. Pearson, and S. D. Bader, Appl. Phys. Lett. 82, 3716 (2003).

${ }^{16}$ M. Rahm, M. Schneider, J. Biberger, R. Pulwey, J. Zweck, and D. Weiss, Appl. Phys. Lett. 82, 4110 (2003).

${ }^{17}$ P. Vavassori, M. Grimsditch, V. Novosad, V. Metlushko, and B. Ilic, Phys. Rev. B 67, 134429 (2003).

${ }^{18}$ U. Welp, V. K. Vlasko-Vlasov, J. M. Hiller, N. J. Zaluzec, V. Metlushko, and B. Ilic, Phys. Rev. B 68, 054408 (2003).

${ }^{19}$ C. A. Ross, Annu. Rev. Mater. Sci. 31, 203 (2001).

${ }^{20}$ K. Yu. Guslienko and K. L. Metlov, Phys. Rev. B 63, 100403 (2001).

${ }^{21}$ K. Yu. Guslienko, V. Novosad, Y. Otani, H. Shima, and K. Fukamichi, Phys. Rev. B 65, 024414 (2001).

${ }^{22}$ Yu. B. Grebenshchikov and N. A. Usov, J. Appl. Phys. 93, 4810 (2003).

${ }^{23}$ J. K. Ha, R. Hertel, and J. Kirschner, Phys. Rev. B 67, 224432 (2003); 67, 064418 (2003).

${ }^{24}$ Yu. B. Grebenshchikov, N. A. Usov, and K. S. Pestchanyi, J. Appl. Phys. 94, 6649 (2003).

${ }^{25}$ The standard Landau approach to study magnetic phase transitions is presented, e.g., in L. D. Landau, E. M. Lifshitz, and L. P. Pitaevskii, Electrodynamics of Continuous Media (ButterworthHeinemann, Oxford 1995).

${ }^{26}$ C. Domb and M. S. Green, Phase Transitions and Critical Phenomena (Academic, London, 1995), Vols. 1-13.

${ }^{27}$ J. J. Binney, N. J. Dowrick, A. J. Fisher, and M. E. J. Newman, The Theory of Critical Phenomena: An Introduction to the Renormalization Group (Clarendon, Oxford, 2002).

${ }^{28}$ G. D'Anna and F. Nori, Phys. Rev. Lett. 85, 4096 (2000).

${ }^{29}$ F. Mertens, S. V. Franklin, and M. Marder, Phys. Rev. Lett. 78, 4502 (1997); S. V. Franklin, F. Mertens, and M. Marder, Phys. Rev. E 62, 8195 (2000).

${ }^{30}$ S. Savel'ev, F. Marchesoni, and F. Nori, Phys. Rev. Lett. 91, 010601 (2003); 92, 160602 (2004).

${ }^{31}$ G. D’Anna, P. Mayor, A. Barrat, V. Loreto, and F. Nori, Nature (London) 424, 909 (2003).

${ }^{32}$ K. Yu. Guslienko, V. Novosad, Y. Otani, H. Shima, and K. Fukamichi, Appl. Phys. Lett. 78, 3848 (2001).

${ }^{33}$ A. Aharoni, J. Appl. Phys. 68, 2892 (1990).

${ }^{34}$ N. A. Usov, S. E. Peschany, J. Magn. Magn. Mater. 118, L290 (1993).

${ }^{35}$ It is more descriptive and accurate to call these spin arrangement as "parallel-spin" state (as opposed to the usual notation: "single-domain" phase) because all spins are parallel to the applied magnetic field.
${ }^{36}$ J. Shibata, K. Shigeto, and Y. Otani, Phys. Rev. B 67, 224404 (2003).

37 W. H. Zurek, Nature (London) 382, 297 (1996); Phys. Rep. 276, 177 (1996).

${ }^{38}$ L. D. Landau and E. M. Lifshitz, Theory of Elasticity (Butterworth-Heinemann, Oxford, 1995).

${ }^{39}$ S. M. Carr and M. N. Wybourne, Appl. Phys. Lett. 82, 709 (2003); S. M. Carr, W. E. Lawrence, and M. N. Wybourne, Phys. Rev. B 64, 220101(R) (2001).

${ }^{40}$ P. Werner and W. Zwerger, Europhys. Lett. 65, 158 (2004).

${ }^{41}$ C. Q. Ru, Phys. Rev. B 62, 16962 (2000); 62, 9973 (2000).

${ }^{42}$ D. Srivastava, M. Menon, and K. Cho, Phys. Rev. B 63, 195413 (2001).

${ }^{43}$ A. Pantano, M. C. Boyce, and D. M. Park, Phys. Rev. Lett. 91, 145504 (2003).

${ }^{44}$ M. Arroyo and T. Belytschko, Phys. Rev. Lett. 91, 215505 (2003).

${ }^{45}$ R. Zandi, J. Rudnick, and R. Golestanian, Phys. Rev. E 67, 021803 (2003); 67, 061805 (2003).

${ }^{46}$ T. Eimüller, M. Scholz, P. Guttmann, M. Köhler, G. Bayreuther, G. Schmahl, P. Fischer, and G. Schütz, J. Appl. Phys. 91, 7334 (2002).

${ }^{47}$ L. Golubovic, D. Moldovan, and A. Peredera, Phys. Rev. E 61, 1703 (2000).

${ }^{48}$ S. F. Mingaleev, Y. B. Gaididei, P. L. Christiansen, and Y. S. Kivshar, Europhys. Lett. 59, 403 (2002).

${ }^{49}$ H. Zhou, Y. Zhang, and Z. Ou-Yang, Phys. Rev. B 62, 1045 (2000).

${ }^{50}$ J. Victor, E. Ben-Haïm, and A. Lesne, Phys. Rev. B 66, 060901 (2002).

${ }^{51}$ M. Marder, E. Sharon, S. Smith, and B. Roman, Europhys. Lett. 62, 498 (2003).

${ }^{52}$ E. Sharon, B. Roman, M. Marder, G.-S. Shin, and H. L. Swinney, Nature (London) 419, 579 (2002).

${ }^{53}$ E. Sharon, M. Marder, and H. L. Swinney, Am. Sci. 92, 254 (2004).

${ }^{54}$ H. Rohrer, Ultramicroscopy 42, 1 (1992); Surf. Sci. 299, 956 (1994); Microelectron. Eng. 32, 5 (1996); 27, 3 (1995).

${ }^{55}$ A. D. Armour, M. P. Blencowe, and K. C. Schwab, Phys. Rev. Lett. 88, 148301 (2002).

${ }^{56}$ M. P. Blencowe and M. N. Wybourne, Appl. Phys. Lett. 77, 3845 (2000).

${ }^{57}$ M. Blencowe, Phys. Rep. 395, 159 (2004).

${ }^{58}$ N. Nishiguchi, Phys. Rev. B 68, 121305 (2003).

${ }^{59} \mathrm{~V}$. Novosad et al. (unpublished).

${ }^{60}$ H. Hoffmann and F. Steinbauer, J. Appl. Phys. 92, 5463 (2002).

${ }^{61}$ J. A. Peacock, Cosmological Physics (Cambridge University Press, Cambridge, 1999).

${ }^{62}$ S. Weinberg, The Quantum Theory of Fields: Modern Applications (Cambridge University Press, Cambridge, 1996).

${ }^{63}$ M. E. Peskin and D. V. Schroeder, An Introduction to Quantum Field Theory (Advanced Book Program, Westview Press, Colorado, 1995).

${ }^{64}$ M. Le Bellac, F. Mortessagne, and G. G. Batrouni, Equilibrium and Non-Equilibrium Statistical Thermodynamics (Cambridge University Press, Cambridge, 2004).

${ }^{65}$ M. F. Laguna, C. A. Balseiro, D. Domínguez, and F. Nori, Phys. Rev. B 64, 104505 (2001).

${ }^{66} \mathrm{I}$. B. Bersuker and V. Z. Pollinger, Vibronic Interactions in Molecules and Crystals (Springer, New York, 1989). 
${ }^{67}$ B. J. Baelus, F. M. Peeters, and V. A. Schweigert, Phys. Rev. B 63, 144517 (2001).

${ }^{68}$ L. F. Chibotaru, A. Ceulemans, V. Bruyndoncx, and V. V. Moshchalkov, Nature (London) 408, 833 (2000); Phys. Rev. Lett. 86, 1323 (2001).

${ }^{69}$ V. R. Misko, V. M. Fomin, J. T. Devreese, and V. V. Moshchalkov, Phys. Rev. Lett. 90, 147003 (2003).
${ }^{70} \mathrm{~K}$. Kadowaki et al. (unpublished).

${ }^{71}$ J. E. Villegas, S. Savel'ev, F. Nori, E. M. Gonzalez, J. V. Anguita, R. García, and J. L. Vicent, Science 302, 1188 (2003).

${ }^{72}$ Y. Jaccard, J. I. Martín, M.-C. Cyrille, M. Vélez, J. L. Vicent, and I. K. Schuller, Phys. Rev. B 58, 8232 (1998).

${ }^{73}$ M. V. Milošević and F. M. Peeters, Physica C 404, 281 (2004); 404, 246 (2004). 